\title{
Endogenous Public Information and Welfare in Market Games*
}

\author{
Xavier Vives
}

IESE Business School

December 2015

\begin{abstract}
This paper performs a welfare analysis of markets with private information in which agents condition on prices in the rational expectations tradition. Price-contingent strategies introduce two externalities in the use of private information: a pecuniary externality and a learning externality. The pecuniary externality induces agents to put too much weight on private information and in the standard case, when the allocation role of the price prevails over its informational role, overwhelms the learning externality which impinges in the opposite way. The price may be very informative but at the cost of an excessive dispersion of the actions of agents. The welfare loss at the market solution may be increasing in the precision of private information. The analysis provides insights into optimal business cycle policy and a rationale for a Tobin-like tax for financial transactions.
\end{abstract}

Keywords: learning externality, asymmetric information, pecuniary externality, team solution, rational expectations, cursed equilibrium, Tobin tax, business cycle policy.

JEL Codes: D82, D83, G14, H23.

* A previous version of the paper was circulated under the title "Endogenous Public Information and Welfare". I am grateful to Marios Angeletos, Anna Bayona, Matthew Gentzkow, Jennifer La'O, Jean-Paul L'Huillier, Carolina Manzano, Manuel Mueller-Frank, Kristoffer Nimark, Morten Olsen, Alessandro Pavan, Venky Venkateswaran, Muhamet Yildiz, the editor Phillipp Kircher, and anonymous referees for providing very useful comments to different versions of the paper, and to Rodrigo Escudero and Jorge Paz for their very able research assistance. The work presented draws also on my early collaboration with Simon Messner. The research leading to these results received funding from the European Research Council under the European Advanced Grants scheme and from project ECO-2011-29533 of the Spanish Ministry of Education. 


\section{Introduction}

We show that when agents can condition on prices the presumption that they will put too little weight on private information need not hold. Agents may put too much weight on private information and prices may contain "too much" information from a second best perspective for reasons other than the well-known Hirshleifer (1971) effect of destruction of insurance opportunities. The results provide a rationalization of pro-cyclical business cycle policy and a Tobin-style tax on financial transactions.

In many markets agents compete in demand and/or supply schedules and therefore condition on prices. In those markets the strategy for an agent is a demand function, as is common in financial markets and in asset auctions such as those of Treasuries or central bank liquidity, or a supply function as in wholesale electricity. In the latter case electricity generators submit a schedule of supply at different prices, the schedules are aggregated by the market mechanism, and a market clearing price is found crossing the aggregate supply schedule with the demand schedule. The production of firms is then contingent on the realized market price. Prices are main providers of endogenous public information. In financial markets, prices are noisy statistics that arise from the decisions of traders and the market clearing mechanism. In goods markets, prices aggregate information about the costs of firms and/or the preferences of consumers and the quality of the products.

The received literature on information externalities points at agents typically relying too much on public information. The reason is that agents do not take into account that their reaction to private information affects the informativeness of public statistics and general welfare. In other words, agents do not internalize an information externality. Pure information externalities will make agents insufficiently responsive to their private information (Vives 1993, 1997; Amador and Weill 2012) and, in the limit to disregard it (Banerjee 1992, Bikhchandani et al. 1992). For example, Morris and Shin (2005) point to the paradox that a central bank by publishing aggregate statistics makes those less reliable by inducing agents in the economy to rely less on their private signals.

In order to speak meaningfully of excessive or insufficient weight to private information, i.e. to perform a welfare analysis in a world with asymmetric information, 
we require a benchmark against which to test market equilibria. An appropriate benchmark for measuring inefficiency at the market equilibrium is the team solution in which agents internalize collective welfare but respecting the decentralized information structure of the economy (Radner 1979; Vives 1988; Angeletos and Pavan 2007). This is in the spirit of Hayek (1945), where the private signals of agents cannot be communicated to a center. The team-efficient solution internalizes the payoff and information externalities associated with the actions of agents in the market. Collective welfare may refer to the surplus of all market participants or may be restricted to the internal welfare of the active agents.

The under-reliance on private information result extends to some classes of economies with endogenous public information. Indeed, consider an economy in which equilibria are team-efficient when public information is exogenous as, for example, a Cournot market with a continuum of firms and private information (Vives 1988). Then increasing public information has to be good marginally, since information is used in a constrained-efficient way, and under regularity conditions the result holds globally. This implies that more weight to private information is needed in relation to the market (Angeletos and Pavan 2009). We show that this logic breaks down in a market game where agents condition on the price, say firms competing in supply functions, because then there is a pecuniary externality related to the use of private information, even if public information were to be exogenous, which makes the market inefficient. The pecuniary externality is that when agents react to their private information they do not take into account that they influence the price, which in turn influences the actions of other agents since they condition on the price. This pecuniary externality may counteract the learning from the price externality and lead agents to put too much weight on private information.

We consider a tractable linear-quadratic-Gaussian model. The context is a market game, where external effects go through the price. There is uncertainty about a common valuation parameter about which agents have private information, and the price is potentially noisy. We use a model with a rational expectations flavor but in the context of a well-specified game where a continuum of agents compete in schedules. We focus our attention on linear Bayesian equilibria. The model is flexible and admits several interpretations in terms of firms competing in a homogenous 
product market (which is the interpretation we follow when developing the model and results) monopolistic competition, and trading in a financial market.

Let us discuss the results in some more detail. For concreteness, consider a homogenous product market with downward sloping demand subject to a random shock and a continuum of ex ante identical firms competing in supply schedules with increasing marginal costs which have an uncertain common level. Each firm receives a private signal as well as a public signal about the uncertain common level (the intercept with the ordinate axis of the marginal cost function).

In the economy considered the full information equilibrium is efficient since it is competitive. In this equilibrium all firms produce the same amount since they all have full information on costs, which are symmetric and strictly convex. In this situation the covariance between the price-cost margin and the cost surprise (that is, the departure of the cost realization from its public expectation) is zero. When firms receive imperfect signals they underreact on average to a cost surprise with respect to the benchmark where firms observe the cost shock. For instance, they produce a relatively high output when the cost shock is high. This raises marginal costs -since marginal costs are increasing in output- and induces a negative covariation between the price-cost margin and the cost surprise. Private information introduces both aggregate and distributive inefficiency. Aggregate inefficiency refers to a distorted total output and distributive inefficiency refers to a distorted distribution of a given total output. The team-efficient solution, preserving the decentralized private information structure of the economy, optimally trades off the tension between the two sources of welfare loss. However, the market does not get it right.

The reason is that conditioning on prices introduces two information-related externalities. The first one, termed pecuniary, arises even if firms do not take into account the information content of prices, i.e., they are naïve as in a fully cursed equilibrium (Eyster and Rabin 2005), and even if there is no noise in demand. There is a pecuniary externality in the use of private information at the (naïve) competitive equilibrium because firms use price-contingent strategies but they do not take into account how their response to private information affects the price. This externality and its effects are novel: it leads firms to put too much weight on their private signals. 
The second externality is the learning externality, by now well understood, which leads firms to underweight private information because firms do not anticipate the influence of their actions on the information content of the price.

The bias that the pecuniary externality introduces in the strategies of firms and in the market solution when there is asymmetric information is subtle. Suppose that firms ignore the information content of the price. Changes in the firms' responsiveness to private information in their output strategy affect the sensitivity of the equilibrium price to the cost surprise. However, this is ignored by the firms when they make their individually optimal choices and it has an external effect because firms condition output decisions on prices. For example, a larger sensitivity of the firm's output strategy to private information makes the equilibrium price more sensitive to the cost surprise. The problem for welfare is that this induces firms to produce too much when costs are high (and margins low) and too little when they are low (and margins high) with respect to the team benchmark. Note that the price-cost margin provides the right welfare indicator in our model with a continuum of firms. The market inefficiency can be corrected by inducing firms to moderate their response to their private signals and making the price less sensitive to cost surprises. The inefficiency does not depend on the level of noise in demand since we have assumed that firms disregard the information content of the price.

Consider now sophisticated firms taking into account the information content of the price. In this case noise in demand avoids the price being fully revealing. In this context, firms correct the slope of their strategy according to what they learn from the price. The price's informational and allocative roles conflict since a high price is bad news (high cost) and the equilibrium supply schedule is steeper than with full information. In fact, in equilibrium schedules may slope downwards when the informational role of prices dominates their allocative role. This will occur when there is little noise in the demand shock and therefore in the price.

We have thus the pecuniary externality leading to over-reliance in private information and the learning externality leading to under-reliance. Depending on the relative strength of the learning externality we may overcome or not the overweighting result due to the pecuniary externality. The point where both externalities cancel each other 
is when firms use vertical supply schedules (as in a Cournot market). In the standard case where supply is upward sloping, which happens when noise in demand is high, the allocative effect of the price prevails and the learning externality is weak. In this case the pecuniary externality effect wins over the learning externality and the weight to private information is too large. The somewhat surprising possibility that prices are "too informative" may arise since even though to have more informative prices is good for aggregate efficiency this comes in a second best world at the cost of increasing dispersion and productive inefficiency. Indeed, to have more informative prices firms have to respond more to their private signals and this magnifies the noise they contain. When the supply function is downward sloping, which happens when noise in demand is low, the informational component of the price prevails and the learning externality is strong. In this case the learning externality wins over the pecuniary externality and the weight to private information is too small.

More precise information, be it public or private, reduces the welfare loss at the teamefficient solution. The reason is that the direct impact of the increased precision is to decrease the welfare loss and this is the whole effect since at the team-efficient solution the responses to private and public information are already (socially) optimized (this is as in Angeletos and Pavan 2009). In contrast, at the market solution an increase in, say, the precision of private information will increase the response of a firm to its private signal and this will tend to increase the welfare loss when the market calls already for a too large response to private information. If this indirect effect is strong enough the welfare loss may be increasing with the precision of private information. In principle the same effect could happen with the precision of public information but we can show that the indirect effect of changes in both the exogenous public precision of information and the precision of the noise in the endogenous public signal are always dominated by the direct effect. The result is that the welfare loss at the market solution is always decreasing with the precisions of public information.

The team-efficient solution can be implemented with tax-subsidy schemes; in particular, with a quadratic transaction tax. This may rationalize a Tobin-like tax in the context of a financial market whenever the allocative role of the price prevails 
over its informational role. ${ }^{1}$ In this case the transaction tax makes informed traders to internalize the pecuniary externality in the use of private information. The end result is a price which contains less information and it may even result in a deeper market.

The results can be extended to the case where the exogenous demand function is upward sloping (as in an addictive good) and to using an internal team-efficient benchmark (where only the collective welfare of the active players is taken into account, for example, ignoring passive consumers). In this latter case the full information market does not achieve an efficient outcome.

The paper follows the tradition of the literature on the welfare analysis of private information economies (Palfrey 1985, Vives 1988, Angeletos and Pavan 2007, 2009), extending the analysis to endogenous public information when the public signal is the price. To do so it builds on the models of strategic competition on schedules such as Kyle (1985) and Vives (2011) but in a continuum-of-agents framework. ${ }^{2} \mathrm{We}$ contribute to the recent surge of interest in the welfare analysis of economies with private information and in particular on the role of public information in such economies (see, e.g., Morris and Shin 2002; Angeletos and Pavan 2007; Amador and Weill 2010). Our results qualify the usual intuition of informational externality models (Vives 1997, Amador and Weill 2010, 2012) in a market game model. It is worth noting that pecuniary externalities are associated with inefficiency in competitive but incomplete markets and/or in the presence of private information since then the conditions of the first fundamental welfare theorem are not fulfilled. Competitive equilibria are not constrained efficient in those circumstances (Greenwald and Stiglitz 1986). ${ }^{3}$ In our paper (as in Laffont 1985) competitive noisy

1 A tax on short-term speculation was proposed by Keynes (1936) and advocated by Tobin (1978) later on with the celebrated phrase "to throw sand in the wheels of the excessively efficient international money markets". The Tobin tax has been advocated by, among others, Stiglitz (1989) and Summers and Summers (1989) who argue for its benefits even if it reduces market liquidity. Taxes of this sort have been in place in several countries (such as the UK and Sweden) and more recently, after the financial crisis, the Financial Transactions Tax (FTT) is on the agenda: 11 European countries have committed to introduce it, with delayed implementation to January $1^{\text {st }}$, 2016, and France has already moved to introduce a version of a FTT in August 2012.

2 Vives (2014) also uses a continuum of agents framework to study the Grossman-Stiglitz paradox in a related model where agents have private valuation utility parameters.

3 For example, pecuniary externalities in markets with financial frictions (borrowing or collateral constraints) can explain market failure (see, e.g., Caballero and Krishnamurthy 2001 and Jeanne and Korinek 2010). 
rational expectations equilibria (REE), in which traders take into account information from prices, are not constrained efficient. ${ }^{4}$ In our quasilinear utility model there is no room for the Hirshleifer (1971) effect according to which REE may destroy insurance opportunities by revealing too much information. We provide therefore an instance of REE which may reveal too much information on a fundamental (from a second best perspective) which is independent of the Hirshleifer effect. ${ }^{5}$

Recent literature has examined the circumstances under which more public information actually reduces welfare (as in Burguet and Vives 2000; Morris and Shin 2002; Angeletos and Pavan 2007; Amador and Weill 2010, 2012). In Burguet and Vives (2000) a higher (exogenous) public precision may discourage private information acquisition and lead to a higher welfare loss in a purely informational externality model. In Morris and Shin (2002) the result is driven by a socially excessive incentive to coordinate by agents. Angeletos and Pavan (2007) qualify this result and relate it to the payoff externalities present in a more general model. In Amador and Weill (2010) a public release of information reduces the informational efficiency of prices and this effect may dominate the direct information provision effect. Their model is purely driven by information externalities in the presence of strategic complementarities in terms of responses to private information. ${ }^{6}$ In our model, which is based on competition on schedules, more public information is not damaging welfare but more private precision may be.

The plan of the paper is as follows. Section 2 presents the model with the leading interpretation of firms competing in a homogenous product market. Section 3 introduces the welfare benchmark. Section 4 characterizes equilibrium and welfare with exogenous public information. Section 5 considers endogenous public information with firms learning from prices. Section 6 considers the case with upward

4 If the signals of agents can be communicated to a center, as in Laffont (1985) then questions arise concerning the incentives to reveal information and how welfare allocations may be modified. This issue is analyzed in a related model by Messner and Vives (2006).

5 See the general analysis of the value of public information in Schlee (2001).

6 In Amador and Weill (2010) there is no direct complementarity or substitutability in actions. However, complementarity or substitutability arises indirectly because workers learn from prices, and the informativeness of prices is affected by the actions of agents. 
sloping (exogenous) demand. Section 7 deals with demand function competition and the role of optimal taxes. Section 8 studies the internal team-efficient benchmark. Concluding remarks are provided in Section 9 and proofs are gathered in the Appendix. Supplementary material, including some more proofs and an analysis of the Cournot case with endogenous public information is provided in the online appendix.

\section{The market game}

Consider a continuum of firms indexed within the interval $[0,1]$ (endowed with the Lebesgue measure), $x_{i}$ is the output of firm $i$, produced at $\operatorname{cost} C\left(x_{i}\right)=\theta x_{i}+(\lambda / 2) x_{i}^{2}$ where $\theta$ is random and $\lambda>0$. Firms face an inverse demand for an homogenous product $p=\alpha+u-\beta \tilde{x}$, where $u \sim N\left(0, \sigma_{u}^{2}\right)$ with $\sigma_{u}^{2} \geq 0$ is a demand shock following a Gaussian distribution; $\alpha>0, \beta>0$, and $\tilde{x}=\int_{0}^{1} x_{i} d i$ is the aggregate output. The demand can be derived from a quadratic utility function from a representative consumer with quasilinear preferences. With decreasing marginal utility we have that $\beta>0$ and demand is downward sloping. The case with $\beta<0$ (upward sloping demand) will be considered in Section 6.

The parameter $\theta$ has prior Gaussian distribution with mean $\bar{\theta}$ and variance $\sigma_{\theta}^{2}>0$ $\left(\theta \sim N\left(\bar{\theta}, \sigma_{\theta}^{2}\right)\right.$ and, to ease notation, set $\left.\bar{\theta}=0\right)$. Firm $i$ receives a private signal $S_{i}=\theta+\varepsilon_{i}$ with $\varepsilon_{i} \sim N\left(0, \sigma_{\varepsilon}^{2}\right), \operatorname{cov}\left[\varepsilon_{i}, \varepsilon_{j}\right]=0, j \neq i$, and a public signal $z=\kappa \theta+\omega$ where $\kappa \geq 0$ and $\omega \sim N\left(0, \sigma_{\omega}^{2}\right) \cdot{ }^{7}$ The error term $\omega$ will be independent of $u$ if the public signal is exogenous and $\omega=u$ if the public signal is endogenous (linked to the market price). In the first case the random variables $\left\{\theta,\left(\varepsilon_{i}\right), u, \omega\right\},\left(\varepsilon_{i}\right) \equiv\left(\varepsilon_{i}\right)_{i \in[0,1]}$, are mutually independent, and in the second $\left\{\theta,\left(\varepsilon_{i}\right), u\right\}$ are. In any case the

7 As an example, the cost parameter $\theta$ could be a unit ex post pollution damage that is assessed on a firm, say an electricity generator, and for which the firm has a private estimate before submitting its supply function. 
information set of firm $i$ is therefore $I_{i}=\left\{s_{i}, z\right\}$. Firms use supply functions as strategies.

Given a random variable $y$ we denote by $\tau_{y} \equiv 1 / \sigma_{y}^{2}$ its precision. We follow the convention that error terms cancel in the aggregate: $\int_{0}^{1} \varepsilon_{i} d i=0$ almost surely (a.s.). ${ }^{8}$ Then the aggregation of all individual signals will reveal the underlying uncertainty: $\int_{0}^{1} s_{i} d i=\theta+\int_{0}^{1} \varepsilon_{i}=\theta .9$

The timing of events is as follows. At $t=0$, the random variables $\theta$ and $u$ are drawn but not observed. At $t=1$, consumers and producers form demand and supply plans. A consumer maximizes utility knowing the realization of $u$. Each firm submits a supply schedule $X_{i}\left(I_{i} ;\right)$ contingent on his information set $I_{i}=\left\{s_{i}, z\right\}$ with $x_{i}=X_{i}\left(I_{i} ; p\right)$ where $p$ is the price. The strategy of a firm is a map from the signal vector space to the space of schedules. At $t=2$ the market clears, the price is formed by finding a $p$ that solves $p=\alpha+u-\beta\left(\int_{0}^{1} X_{j}\left(I_{j} ; p\right) d j\right)$. Finally, consumption occurs and payoffs are collected.

Let us assume that there is a unique price $\hat{p}\left(\left(X_{j}\left(I_{j} ; \cdot\right)\right)_{j \in[0,1]}\right)$ for any realization of the signals. ${ }^{10}$ Then, for a given profile $\left(X_{j}\left(I_{j} ; \cdot\right)\right)_{j \in[0,1]}$ of firms' schedules and realization of the signals, the profits for firm $i$ are given by

8 Equality of random variables has to be understood to hold almost surely. We will not insist on this in the paper.

9 Suppose that $\left(q_{i}\right)_{i \in[0,1]}$ is a process of independent random variables with means $\mathbb{E}\left[q_{i}\right]$ and uniformly bounded variances $\operatorname{var}\left[q_{i}\right]$. Then we let $\int_{0}^{1} q_{i} d i=\int_{0}^{1} \mathbb{E}\left[q_{i}\right] d i$ (a.s.). This convention will be used while taking as given the usual linearity property of integrals. (Equality of random variables is assumed to hold almost surely always.) In short, we assume that the strong law of large numbers (SLLN) holds for a continuum of independent random variables with uniformly bounded variances.

10 We assign zero payoffs to the players if there is no $p$ that solves the fixed point problem. If there are multiple solutions, then the one that maximizes volume is chosen. 


$$
\pi_{i}=(p-\theta) x_{i}-\frac{\lambda}{2} x_{i}^{2}
$$

where $x_{i}=X_{i}\left(I_{i} ; p\right)$, and $p=\hat{p}\left(\left(X_{j}\left(I_{j} ; \cdot\right)\right)_{j \in[0,1]}\right)$. This defines a Bayesian game in schedules. If the public signal $z$ in $I_{i}=\left\{s_{i}, z\right\}$ is exogenous then the firms are "naïve" and do not take into account the information content of the price. If the public signal is endogenous (the price) then firms are sophisticated and the formulation has a rational expectations flavor but in the context of a well-specified schedule game. In this second case we will restrict our attention to linear Bayesian equilibria of the schedule game. ${ }^{11}$

It is worth to remark that in the market game with sophisticated firms both payoff and learning externalities go through the market price $p$, which has both an allocative and an informational role. When $\beta=0$, the price is independent of $\tilde{x}$ and there are neither payoff nor informational externalities among players.

The model admits other interpretations: exogenous upward sloping demand $(\beta<0$, Section 6), demand function competition (Section 7), or monopolistic competition (Section 8). ${ }^{12}$

We will study Bayesian equilibria of the supply function game in two versions. In the first firms will be "naïve" in the sense of not taking into account the information content of the price and in the second they will. In the first therefore the public signal $z$ will be exogenous while in the second it will reflect the information content of the price. In the exogenous information case noise in demand plays no special role and we can have $\sigma_{u}^{2}=0$ while when there is learning from the price we need $\sigma_{u}^{2}>0$ to avoid prices being fully revealing of $\theta$. Before that in the next section we define the appropriate welfare benchmark for the use of information in our economy.

11 Normality of random variables means that prices and quantities can be negative with positive probability. The probability of this event can be controlled, if necessary, by an appropriate choice of means and variances. Furthermore, for this analysis the key property of Gaussian distributions is that conditional expectations are linear. Other prior-likelihood conjugate pairs (e.g., beta-binomial and gamma-Poisson) share this linearity property and can display bounded supports.

12 See also Chapter 3 in Vives (2008) for an overview of the connection between supply function competition and rational expectations models, as well as examples. 


\section{The welfare benchmark}

Consider an allocation assigning output $x_{i}$ to firm $i$ and with average output $\tilde{x}$. We will use a utilitarian welfare criteria, which in our quasilinear world is equivalent to total surplus:

$$
\mathrm{TS} \equiv\left(\alpha+u-\beta \frac{\tilde{x}}{2}\right) \tilde{x}-\int_{0}^{1}\left(\theta x_{i}+\frac{\lambda}{2} x_{i}^{2}\right) d i
$$

It is immediate that the first-best (full information) allocation has all firms producing the same amount, $x^{o}=(\beta+\lambda)^{-1}(\alpha+u-\theta) \cdot{ }^{13}$ Denote by $\mathrm{TS}^{o}$ total surplus at the full information first best. The first-best allocation is attained by the competitive market when there is full information (i.e., firms receive perfect signals about $\theta$ ). The market equilibria we consider under asymmetric information will not attain in general the first best. The reason is that suppliers produce under uncertainty and rely on imperfect idiosyncratic estimation of the common cost component; hence they end up producing different amounts even though costs are identical and strictly convex.

Using the expression for first best output $x^{o}$ we have that for a symmetric allocation for which $\left.\mathbb{E}\left[\int_{0}^{1}\left(x_{i}-\tilde{x}\right)^{2} d i\right]=\mathbb{E}\left[\left(x_{i}-\tilde{x}\right)^{2}\right]\right), \mathbb{E}[\mathrm{TS}]=\mathbb{E}\left[\mathrm{TS}^{\circ}\right]-\mathrm{WL}$ where WL is the expected welfare loss:

$$
\mathrm{WL}=\left((\beta+\lambda) \mathbb{E}\left[\left(\tilde{x}-x^{o}\right)^{2}\right]+\lambda \mathbb{E}\left[\left(x_{i}-\tilde{x}\right)^{2}\right]\right) / 2 .
$$

The first term in WL corresponds to aggregate inefficiency (how distorted is the average quantity $\tilde{X}$ while producing in a cost-minimizing way), which is proportional to $\mathbb{E}\left[\left(\tilde{x}-x^{o}\right)^{2}\right]$, and the second term to distributive inefficiency (how distorted is the distribution of production of a given average quantity $\tilde{X}$ ), which is proportional to the dispersion of outputs $\mathbb{E}\left[\left(x_{i}-\tilde{x}\right)^{2}\right]$. Let $p^{o}$ be the full information first best price. Note that $\mathbb{E}\left[\left(p-p^{o}\right)^{2}\right]=\beta^{2} \mathbb{E}\left[\left(\tilde{x}-x^{o}\right)^{2}\right]$.

\footnotetext{
13 Note that TS is strictly concave for symmetric solutions since $\beta+\lambda>0$.
} 
The welfare benchmark we use is the team solution maximizing expected total surplus subject to employing linear decentralized strategies (as in Vives 1988; Angeletos and Pavan 2007). This team-efficient solution internalizes the payoff and information externalities of the actions of agents, and it is restricted to using the same type of strategies (decentralized and linear) that the market employs. The question then is whether the team solution can strictly improve upon the market allocation.

It is worth noting that in the economy considered if firms were not to condition on prices, i.e. if each firm would set quantities as strategies, conditioning only on its available signals as in a Cournot market, instead of using a supply function, then the market solution would be team-efficient (Vives 1988, see Section 4.3). That is, in the Cournot economy, the private information equilibrium is team-efficient for given public information. We will see that this is not the case in our market game with price-contingent strategies even when firms disregard the information content of the price (i.e. with exogenous public information) because of a pecuniary externality in the use of private information.

\section{Equilibrium and welfare with exogenous public information}

In this section we consider the case in which firms are naïve in the sense that they do not realize the informational value of the price (i.e., there is no learning from the price). This situation corresponds to the case of fully cursed equilibrium of Eyster and Rabin (2005). Indeed, in a fully cursed equilibrium each player assumes no connection between the actions and types of other players. In this case the price is perceived to have no information about the parameter $\theta$ and the information set of firm $i$ is $I_{i}=\left\{s_{i}, z\right\}$ where $z=\kappa \theta+\omega$ and $u$ and $\omega$ are independent.

\subsection{Equilibrium}

At the market equilibrium, firm $i$ solves

$$
\max _{x_{i}}\left(p-\mathbb{E}\left[\theta \mid s_{i}, z\right]-\frac{\lambda}{2} x_{i}\right) x_{i} .
$$

The solution is both unique (given strict concavity of profits) and symmetric across firms (since the cost function and signal structure are symmetric across firms): 


$$
X\left(s_{i}, z ; p\right)=\lambda^{-1}\left(p-\mathbb{E}\left[\theta \mid s_{i}, z\right]\right)
$$

Let $\hat{z}=\mathbb{E}[\theta \mid z]$ denote the public expectation of $\theta$. From the projection theorem for Gaussian random variables we have that $\mathbb{E}\left[\theta \mid s_{i}, z\right]=\gamma s_{i}+(1-\gamma) \hat{z}$, where the weight to private information $\gamma=\tau_{\varepsilon}\left(\tau_{\varepsilon}+\tau\right)^{-1}$ is the Bayesian weight, with $\tau \equiv(\operatorname{var}[\theta \mid z])^{-1}=\tau_{\theta}+\tau_{\omega} \kappa^{2} .14$ Averaging the demand of firms we obtain $\tilde{x}=p-(a \theta+(1-a) \hat{z})$, and from market clearing, $p=\alpha+u-\beta \tilde{x}$, it is immediate that the equilibrium is unique, symmetric and linear. Let $a \equiv-\partial x_{i} / \partial s_{i}=\lambda^{-1} \gamma$ denote the response to private information in the strategy of firm $i$.

\subsection{Team solution}

At the team-efficient solution with exogenous public information expected total surplus $\mathbb{E}[\mathrm{TS}]$ is maximized subject to firms using decentralized linear (affine) production strategies contingent on their information set $I_{i}=\left\{s_{i}, z\right\}$ and on the price (but with no learning from it; i.e., perceiving no covariance between $\theta$ and the price, $\mathbb{E}[\theta p]=0)$. That is, the team solution solves the program:

$$
\max _{a, \hat{b}, c, \hat{c}} \mathbb{E}[\mathrm{TS}] \quad\left(\mathbf{T}_{\text {exo }}\right)
$$

subject to $x_{i}=\hat{b}-a s_{i}-c z+\hat{c} p, \quad p=\alpha+u-\beta \tilde{x}, \mathbb{E}[\theta p]=0$, and $z=\kappa \theta+\omega$.

It can be shown (see Claim 1 in the Appendix) then that team strategy is of the same form as in the market solution, $X\left(s_{i}, z ; p\right)=\lambda^{-1}\left(p-\left(\gamma s_{i}+(1-\gamma) \hat{z}\right)\right)$, but where now the weight to private information $\gamma$ may differ from the Bayesian weight. At a candidate team strategy public information is optimally used for a given weight to private information, i.e. the weights to private and public information $(\hat{z}=\mathbb{E}[\theta \mid z])$ have to add up to one. We will see that the team solution can improve upon the

14 Indeed, $\gamma$ minimizes the mean square error of predicting $\theta$ with the private and public signals

$$
I_{i}=\left\{s_{i}, z\right\}: \min _{\gamma} \frac{1}{2 \lambda}\left(\frac{(1-\gamma)^{2}}{\tau}+\frac{\gamma^{2}}{\tau_{\varepsilon}}\right) \text {. }
$$


market even when restricting strategies to those with the same form as in the market solution.

Averaging the candidate equilibrium strategy and using the market clearing condition we obtain that $\tilde{x}=(\alpha+u-(\lambda a \theta+(1-\lambda a) \hat{z})) /(\beta+\lambda)$. It follows that the welfare loss at any candidate team solution will depend only on the response to private information $a$. Since $x^{o}=(\beta+\lambda)^{-1}(\alpha+u-\theta)$ we obtain that $\mathbb{E}\left[\left(\tilde{x}-x^{o}\right)^{2}\right]=(1-\lambda a)^{2} /\left(\tau(\beta+\lambda)^{2}\right)$ and $\mathbb{E}\left[\left(x_{i}-\tilde{x}\right)^{2}\right]=a^{2} \sigma_{\varepsilon}^{2}$, and from the expression of the welfare loss in Section 3 we have that

$$
\mathrm{WL}(a ; \tau)=\frac{1}{2}\left(\frac{(1-\lambda a)^{2}}{\tau(\beta+\lambda)}+\frac{\lambda a^{2}}{\tau_{\varepsilon}}\right),
$$

where $\tau=\tau_{\theta}+\tau_{\omega} \kappa^{2}$, which is easily seen strictly convex in $a$. Changing $a$ has opposite effects on both sources of the welfare loss since aggregate inefficiency decreases with $a$, as the average quantity gets close to the full information allocation, but distributive inefficiency increases with $a$ as dispersion increases.

The team solution for given exogenous public precision $\tau$, denoted $a_{\mathrm{exo}}^{\mathrm{T}}(\tau)$, minimizes WL and optimally trades off the sources of inefficiency among decentralized strategies. It is worth noting that WL is independent of $\sigma_{u}^{2}$ and therefore $a_{\mathrm{exo}}^{\mathrm{T}}(\tau)$ will also be independent of $\sigma_{u}^{2}$. We obtain that $a_{\mathrm{exo}}^{\mathrm{T}}(\tau)=\tau_{\varepsilon}\left(\lambda\left(\tau_{\varepsilon}+\tau\right)+\beta \tau\right)^{-1}$. The market solution is given by $a_{\text {exo }}^{*}(\tau)=\tau_{\varepsilon}\left(\lambda\left(\tau_{\varepsilon}+\tau\right)\right)^{-1}$. It follows that $a_{\text {exo }}^{\mathrm{T}}(\tau)<a_{\text {exo }}^{*}(\tau)$ if and only if $\beta>0$. The following proposition states the result.

Proposition 1. Let $\infty>\tau_{\varepsilon}>0, \beta>0$, and $\sigma_{u}^{2} \geq 0$, suppose that firms receive a public signal of precision $\tau$ and ignore the information content of the price. Then, at the unique equilibrium, firms respond more to private information $a_{\mathrm{exo}}^{*}(\tau)$ than at the team optimal solution $a_{\mathrm{exo}}^{\mathrm{T}}(\tau)$ : 


$$
a_{\mathrm{exo}}^{*}(\tau)=\frac{\tau_{\varepsilon}}{\lambda\left(\tau_{\varepsilon}+\tau\right)}>a_{\mathrm{exo}}^{\mathrm{T}}(\tau)=\frac{\tau_{\varepsilon}}{\lambda\left(\tau_{\varepsilon}+\tau\right)+\beta \tau}
$$

Three remarks are in order.

(i) Noise in demand plays no role in the result (i.e. the proposition holds for $\left.\sigma_{u}^{2}=0\right)$.

(ii) Inefficiency arises because of asymmetric information when $\beta>0$. Indeed, if $\tau_{\varepsilon}=0$, then $a_{\text {exo }}^{*}(\tau)=a_{\text {exo }}^{\mathrm{T}}(\tau)=0$ and if $\tau_{\varepsilon} \rightarrow \infty$, then both tend to 1 . When information is symmetric (there is no private information, $\tau_{\varepsilon}=0$, or information is perfect, $\tau_{\varepsilon}=\infty$ ) the market is efficient since it is competitive and pecuniary externalities are internalized.

(iii) The market and team solutions coincide when $\beta=0$ (i.e. when the price is independent of aggregate output and therefore of the cost shock $\theta$ ). In this case there no externalities whatsoever.

With asymmetric information, the team solution depends indeed on $\beta$, with the term $\beta \tau$ reflecting a pecuniary externality at the market solution. There is a pecuniary externality in the use of private information at the (naïve) competitive equilibrium because firms use price-contingent strategies but they do not take into account how their response to private information affects the equilibrium price and therefore the average quantity.

The result is that the market $\tilde{x}$ overreacts to $\theta$, for a given public signal since $a_{\mathrm{exo}}^{*}>a_{\mathrm{exo}}^{\mathrm{T}}$. Indeed, from the expression for $\tilde{x}$ we obtain that $\partial \tilde{x} / \partial \theta=-\lambda a /(\beta+\lambda)$. Note, however, that both the market and the team solution underreact in relation to the first best with full information since $\partial x^{o} / \partial \theta=-1 /(\beta+\lambda)$, and for both market and team solutions $\lambda a<1$. This is so since with noisy signals firms underreact on average to movements in the cost parameter $\theta$. For example, if costs are higher than the public 
expectation of $\theta, \hat{z}=\mathbb{E}[\theta \mid z], \theta-\hat{z}>0$, firms on average produce more than if they knew the cost shock: $\tilde{x}-x^{o}=(1-\lambda a)(\theta-\hat{z}) /(\beta+\lambda)>0 .{ }^{15}$

The presence of the pecuniary externality because firms condition on the price (independently of the information content of the price) is clear but the bias it introduces in the market solution is much more subtle. In order to understand the bias induced by the pecuniary externality in the use of private information generated from conditioning on the price when $\beta \neq 0$ let us consider the effect of a change in the response to private information $a$ at the market solution $a_{\mathrm{exo}}^{*}(\tau)$. From the candidate strategy for firm $i, x_{i}=\lambda^{-1}\left(p-\left(\lambda a s_{i}+(1-\lambda a) \hat{z}\right)\right)$, we have that

$$
\frac{\partial x_{i}}{\partial a}=\left.\frac{\partial x_{i}}{\partial a}\right|_{p}+\frac{\partial x_{i}}{\partial p} \frac{\partial p}{\partial a}
$$

and it is not difficult to check (see proof of Lemma in the appendix A.1) that the margin $\left(p-\operatorname{MC}\left(x_{i}\right)\right)$ gives the right signal for welfare and

$$
\frac{\partial \mathbb{E}[\mathrm{TS}]}{\partial a}=\mathbb{E}\left[\left.\left(p-\operatorname{MC}\left(x_{i}\right)\right) \frac{\partial x_{i}}{\partial a}\right|_{p}\right]+\mathbb{E}\left[\left(p-\operatorname{MC}\left(x_{i}\right)\right) \frac{\partial x_{i}}{\partial p} \frac{\partial p}{\partial a}\right] .
$$

The first term is what the market equates to zero, since firms take as given the price, and the second corresponds to the pecuniary externality in the use of private information since firms do not take into account that they influence the price when they change their response to private information. We can sign the pecuniary externality in two steps.

(1) Note first that at the market solution (first order condition for a firm),

$$
\mathbb{E}\left[\left.\left(p-\operatorname{MC}\left(x_{i}\right)\right) \frac{\partial x_{i}}{\partial a}\right|_{p}\right]=\mathbb{E}\left[\left(p-\operatorname{MC}\left(x_{i}\right)\right)\left(-\left(s_{i}-\hat{z}\right)\right)\right]=0
$$

since $\partial x_{i} /\left.\partial a\right|_{p}=-\left(s_{i}-\hat{z}\right)$. That is, firms in equilibrium eliminate the covariation between the margin $p-\operatorname{MC}\left(x_{i}\right)$ and the cost surprise in relation to the private signal $\left(s_{i}-\hat{z}\right)$ (i.e., the difference between the signal, $s_{i}$, and the public expectation of the

15 Note that since firms are competitive they will produce in expected value the right amount at the equilibrium: $\mathbb{E}[\tilde{x}]=\mathbb{E}\left[x^{\circ}\right]$ (given that $\mathbb{E}[\theta-\mathbb{E}[\theta \mid z]]=0$ ). 
cost parameter, $\hat{z})$. However, if signals were perfect $\left(\sigma_{\varepsilon}^{2}=0\right.$ and $s_{i}=\theta$, a.s. $)$ we would have $\mathbb{E}\left[\left(p-\operatorname{MC}\left(x_{i}\right)\right)(\theta-\hat{z})\right]=0$. That is, firms with full information in equilibrium would eliminate the covariation between the margin $p-\operatorname{MC}\left(x_{i}\right)$ and the cost surprise in relation to the true cost parameter $(\theta-\hat{z})$. The important implication of equilibrium with noisy signals $\left(\sigma_{\varepsilon}^{2}>0\right)$ is that the margin $\left(p-\operatorname{MC}\left(x_{i}\right)\right)$ covaries negatively with $(\theta-\hat{z})$, i.e.,

$$
\mathbb{E}\left[\left(p-\operatorname{MC}\left(x_{i}\right)\right)(\theta-\hat{z})\right]<0 .
$$

That is, in equilibrium, a high cost realization tends to go together with a lower margin. The explanation is as follows. Since there is noise in the signal, a firm will underreact to changes in $\theta$ with respect to the full information benchmark. For example, when the cost realization is high, $(\theta-\hat{z})>0$, a firm will produce more on average than the full information benchmark, and marginal costs $\mathrm{MC}\left(x_{i}\right)$ will increase since the cost function is convex. A high average production pushes the price down according to the inverse demand schedule. The result is that a negative correlation between $p-\operatorname{MC}\left(x_{i}\right)$ and $(\theta-\hat{z})$ is induced at the market equilibrium. ${ }^{16}$

(2) We can sign now the pecuniary externality term. From the market clearing condition $p=\alpha+u-\beta \tilde{x}$ and the expression for the candidate $\tilde{x}$ we obtain

$$
p=((\alpha+u) \lambda+\beta(\lambda a(\theta-\hat{z})+\hat{z}))(\beta+\lambda)^{-1} .
$$

A positive cost surprise, $(\theta-\hat{z})>0$, decreases average supply and increases the equilibrium price. Indeed, the price is increasing in $(\theta-\hat{z})$ given that $\beta>0$. However, since supply is increasing in the price $\left(\partial x_{i} / \partial p=\lambda^{-1}\right)$ an increase in the cost

16 An alternative and equivalent explanation of the negative covariation is the following. In equilibrium the margin will be positively correlated with the error term in the signal of the firm. This is so since for a given cost realization $\theta$, a high signal (i.e. with a positive error term $\varepsilon_{i}>0$ ) will induce firm $i$ to produce less and marginal cost will be low since costs are convex, but the price is not affected by an individual signal (since error terms wash out in the aggregate) and therefore the margin will be high. A positive correlation of the margin and the error term in the signal implies a negative correlation between the margin and the cost surprise since both have to add up to zero. 
surprise cost $(\theta-\hat{z})$ induces via the price channel, and independently of any information contained in the price, an increase in individual supply. Furthermore, a larger response to private information $a$ leads to a decreased production and higher price if the cost surprise is positive $(\theta-\hat{z})>0$, and the opposite if the cost surprise is negative $(\theta-\hat{z})<0$ :

$$
\operatorname{sgn}\{\partial p / \partial a\}=\operatorname{sgn}\{\beta(\theta-\hat{z})\} .
$$

Indeed, a larger reaction to information when costs are high decreases production $(\partial \tilde{x} / \partial a<0)$ and increases the price since the price is decreasing in aggregate supply according to the demand schedule. Now, as explained, the price increase will lead mechanically to a higher output for the firm $x_{i}$ since the supply function is upward sloping, $\partial x_{i} / \partial p=\lambda^{-1}$. The problem is that this is bad for expected profits and welfare since output will be high when the margin is low because of the negative covariance between margins and costs in equilibrium (i.e. $\left.\mathbb{E}\left[\left(p-\operatorname{MC}\left(x_{i}\right)\right)(\theta-\hat{z})\right]<0\right)$.

We obtain the effect of the pecuniary externality for given public information at the market solution for $\beta>0$ :

$$
\operatorname{sgn}\left\{\mathbb{E}\left[\left(p-\operatorname{MC}\left(x_{i}\right)\right) \frac{\partial x_{i}}{\partial p} \frac{\partial p}{\partial a}\right]\right\}=\operatorname{sgn}\left\{\beta \mathbb{E}\left[\left(p-\operatorname{MC}\left(x_{i}\right)\right)(\theta-\hat{z})\right]\right\}<0 .
$$

The following Lemma states the result and provides an explicit expression (with complete proof in the Appendix).

Lemma: At the market solution for given public precision $\tau$ we have that

$$
\left.\frac{\partial \mathbb{E}[\mathrm{TS}]}{\partial a}\right|_{a=a_{e \times 0}^{*}}=\mathbb{E}\left[\left.\left(p-\operatorname{MC}\left(x_{i}\right)\right) \frac{\partial x_{i}}{\partial p} \frac{\partial p}{\partial a}\right|_{a=a_{e x o}^{*}}\right]=-\beta \lambda(\beta+\lambda)^{-1} a_{e \times 0}^{*} \sigma_{\varepsilon}^{2} \cdot{ }^{17}
$$

In summary, the drivers of the pecuniary externality are as follows. A larger sensitivity of the output strategy of a firm to private information (i.e. a larger $a$ ) makes the equilibrium price more sensitive to the cost surprise $\theta-\hat{z}$, but this is

17 And, in fact, the result of Proposition 1 follows since $\mathbb{E}[\mathrm{TS}]$ is strictly concave in $a$. 
ignored by the market (i.e. by the firms when they make their individually optimal choices). This matters for welfare because it induces firms to produce more at the wrong times. Say that the cost surprise is positive $(\theta-\hat{z}>0)$ then an increase in the response to private information $a$ raises the price $p$ since by reacting more strongly to bad news production decreases and the price is decreasing in aggregate supply. This price increase will lead to a higher output $x_{i}$ since the supply function is upward sloping. However, this is inefficient since when costs are high the price-cost margin tends to be low in the market equilibrium. This is bad for expected profits and for welfare since the margin provides the right signal to produce in our competitive economy. The result is that the market puts too much weight on private information and the aggregate quantity overreacts to $\theta$ in relation to the constrained efficient (team) benchmark.

\subsection{Comparison with Cournot competition}

In this section we show that if firms were to compete à la Cournot by setting quantities contingent only on their information then the market solution would be team-efficient. In this case a strategy for firm $i$ is a mapping from signals $I_{i}=\left\{s_{i}, z\right\}$ into outputs: $X_{i}\left(s_{i}, z\right)$. This is the model considered in Vives (1988) (and Angeletos and Pavan, Section 6.1, 2007). ${ }^{18}$ The equilibrium follows immediately from the optimization problem of firm $i, \max _{x_{i}} \mathbb{E}\left[\pi_{i} \mid s_{i}, z\right]$. Since $p=\alpha+u-\beta \tilde{x}$, the associated FOC (which are also sufficient) are $\mathbb{E}\left[p-\operatorname{MC}\left(x_{i}\right) \mid s_{i}, z\right]=0$, where the difference from our market game is that firms do not condition on the price. It follows that, under the same distributional assumptions as in Section 2, there is a unique Bayesian Cournot equilibrium and it is symmetric and linear:

$$
X\left(s_{i}, z\right)=(\beta+\lambda)^{-1}\left(\alpha-\left((\beta+\lambda) a s_{i}+(1-(\beta+\lambda) a) \hat{z}\right)\right)
$$

where $a=\frac{\tau_{\varepsilon}}{\lambda\left(\tau+\tau_{\varepsilon}\right)+\beta \tau_{\varepsilon}}$. It is worth noting that here $(\beta+\lambda) a$ corresponds to the Bayesian weight to private information in $\mathbb{E}\left[\theta \mid s_{i}, z\right]$ only if $\beta=0$.

18 The profits of firm $i$ are $\pi_{i}=(\alpha+u-\beta \tilde{x}) x_{i}-C\left(x_{i}\right)$. Note that in the game in outputs, we have that $\partial^{2} \pi / \partial x_{i} \partial \tilde{x}=-\beta$, and strategies are strategic substitutes with $\beta>0$. 
The market solution is team efficient since the same FOC hold also for the maximization of $\mathbb{E}[\mathrm{TS}]$ subject to decentralized production strategies. Indeed, under our assumptions, it is easily seen that the solution is symmetric and with the same FOC as the market

$$
\mathbb{E}\left[\frac{\partial \mathrm{TS}}{\partial x_{i}} \mid s_{i}, z\right]=\mathbb{E}\left[p-M C\left(x_{i}\right) \mid s_{i}, z\right]=0 .
$$

In the terminology of Angeletos and Pavan (2007), the economy in which agents use non-price contingent strategies displays exactly the right degree of coordination or complementarity. The difference between the Cournot and the supply function mechanism can be seen easily noting that the candidate team strategies with Cournot strategies are of the same form as the market but again with potentially a different response $a$ to private information. At the team optimum we have that:

$$
\frac{\partial \mathbb{E}[\mathrm{TS}]}{\partial a}=\mathbb{E}\left[\left(p-\operatorname{MC}\left(x_{i}\right)\right) \frac{\partial x_{i}}{\partial a}\right]=\mathbb{E}\left[\left(p-\operatorname{MC}\left(x_{i}\right)\right)\left(-\left(s_{i}-\hat{z}\right)\right)\right]=0 .
$$

This is exactly the same FOC than at the market solution where firms maximize expected profits. In contrast, with supply function competition firms condition on the price and do not take into account the pecuniary externality term $\mathbb{E}\left[\left(p-\operatorname{MC}\left(x_{i}\right)\right) \frac{\partial x_{i}}{\partial p} \frac{\partial p}{\partial a}\right]$ analyzed in the previous section.

\section{Equilibrium and welfare with endogenous public information}

We consider now the case where firms are sophisticated and do take into account the information content of prices.

\subsection{Equilibrium}

Suppose that firms receive no public signal except for the price. That is, the information set available to firm $i$ is $\left\{s_{i}, p\right\}$. We are interested in a linear (Bayesian) equilibrium-equilibrium, for short - of the schedule game for which the public statistic functional is of type $P(\theta, u)$. Since the payoffs and the information structure are symmetric and since payoffs are strictly concave, there is no loss of generality in restricting our attention to symmetric equilibria. Indeed, the solution to the problem of firm $i$, 


$$
\max _{x_{i}} \mathbb{E}\left[\left(p-\theta-\frac{\lambda}{2} x_{i}\right) x_{i} \mid s_{i}, p\right],
$$

is both unique (given strict concavity of profits) and symmetric across firms (since the cost function and signal structure are symmetric across firms):

$$
X\left(s_{i}, p\right)=\lambda^{-1}\left(p-\mathbb{E}\left[\theta \mid s_{i}, p\right]\right),
$$

where $p=P(\theta, u)$. Now the estimation of $\theta$ is done with the public endogenous signal $p$. In a linear equilibrium, with responsiveness $a$ to private information, it is easy to see from market clearing that the price $p$ is linearly related to the random variable $z=\kappa \theta+\omega$, with $\kappa=\beta a$ and $\omega=u$. The variable $z$ is informationally equivalent to the price $p$ and $\mathbb{E}\left[\theta \mid s_{i}, p\right]=\mathbb{E}\left[\theta \mid s_{i}, z\right]$. Because $u$ is random, $z$ (and the price) will typically generate a noisy signal of the unknown parameter $\theta$. Let $\tau$ denote the precision of the price $p$ or of $z$ in the estimation of $\theta, \tau \equiv(\operatorname{var}[\theta \mid z])^{-1}$. From the properties of Gaussian random variables it is immediate that $\tau=\tau_{\theta}+\tau_{u} \beta^{2} a^{2}$. The precision $\tau$ of the public signal is now endogenous and increases with the responsiveness to private information $a$.

The information available to firm $i$ is $\left\{s_{i}, p\right\}$ or, equivalently, $I_{i}=\left\{s_{i}, z\right\}$. We can write the strategy of the firm as $X\left(I_{i} ; p\right)=p-\mathbb{E}\left[\theta \mid I_{i}\right]$. Since $\mathbb{E}\left[\theta \mid s_{i}, p\right]=\mathbb{E}\left[\theta \mid s_{i}, z\right]$, with some abuse of notation, we can posit linear strategies of the form $X\left(s_{i}, z\right)=b-a s_{i}+c z$ and solve for the linear equilibrium in the usual way: identifying coefficients with the candidate linear strategy by calculating $\mathbb{E}\left[\theta \mid s_{i}, z\right]$ and using the supply function of a firm. In equilibrium, firms take public information $z$, with precision $\tau \equiv(\operatorname{var}[\theta \mid z])^{-1}$, as given and use it to form probabilistic beliefs about the underlying uncertain parameter $\theta$. This fact is at the root of a learning externality: firms fail to account for the impact of their own actions on public information (the price) and hence on other firms. As in Section 4.1 the market chooses the response to private information in a Bayesian way, $a=\lambda^{-1} \tau_{\varepsilon}\left(\tau_{\varepsilon}+\tau\right)^{-1}$. 
The following proposition characterizes the equilibrium (see Proposition 2A in appendix A.2 for a complete characterization of the equilibrium and its comparative statics properties as well as proofs).

Proposition 2. Let $\tau_{\varepsilon} \geq 0$ and $\tau_{u} \geq 0$. There is a unique (and symmetric) equilibrium

$$
X\left(s_{i}, p\right)=\lambda^{-1}\left(p-\mathbb{E}\left[\theta \mid s_{i}, p\right]\right)
$$

where $a \equiv-\partial x_{i} / \partial s_{i}$ is the unique (real) solution of the equation $a=\lambda^{-1} \tau_{\varepsilon}\left(\tau_{\varepsilon}+\tau\right)^{-1}$ with $\tau=\tau_{\theta}+\tau_{u} \beta^{2} a^{2}$. In equilibrium, a decreases with $\tau_{u}, \tau_{\theta},|\beta|$ and $\lambda$, and increases with $\tau_{\varepsilon}$.

Remark 1. We have examined linear equilibria of the schedule game for which the price functional is of type $P(\theta, u)$. In fact, these are the linear equilibria in strategies with bounded means and with uniformly (across players) bounded variances. (See Claim A in the online appendix.)

Remark 2. It is possible to show that the equilibrium in the continuum economy is the limit of equilibria in replica economies that approach the limit economy. Take the market with a finite number of firms $n$ and inverse demand $p_{n}=\alpha+u-\beta \tilde{x}_{n}$, where $\tilde{x}_{n}$ is the average output per firm, and with the same informational assumptions. In this case, given the results in Section 5.2 of Vives (2011), the supply function equilibrium of the finite $n$-replica market converges to the equilibrium in Proposition 2.

The price serves a dual role as index of scarcity and conveyor of information. Indeed, a high price has the direct effect of increasing a firm's competitive supply, but it also conveys news about costs - namely, that costs are high. Consider as a benchmark the full information case with perfectly informative signals $\left(\tau_{\varepsilon}=\infty\right)$. This is a full information competitive equilibrium and we have $a=\lambda^{-1}$, and $X(\theta, p)=\lambda^{-1}(p-\theta)$. 
In this case, agents have nothing to learn from the price. ${ }^{19}$ If signals become noisy $\left(\tau_{\varepsilon}<\infty\right)$ then $a<\lambda^{-1}$, and supply functions are steeper than with full information as agents correct for the informational content of the price.

As $\tau_{u}$ tends to $\infty$, the precision of prices $\tau$ also tends to $\infty$, the response to private information $a$ tends to 0 , and the equilibrium collapses. Indeed, the equilibrium becomes fully revealing and is not implementable. The informational component of the price increases with $\tau_{u}$ and decreases with $\tau_{\theta}$ (since firms are endowed with better prior information with a larger $\tau_{\theta}$ ). As $\tau_{u}$ increases the slope of supply increases because of the price's increased informational component (a high price indicates higher costs). As $\tau_{u}$ increases more, the slope becomes vertical at some point and then turns negative. ${ }^{20}$ At the point where the allocative and informational effects balance, agents place zero weight on the price and the model reduces to a quantity-setting model à la Cournot (however, not reacting to the price is optimal). If $\tau_{\theta}$ increases then the informational component of the price diminishes and we have a more elastic supply. Market depth $(\partial P / \partial u)^{-1}$ is decreasing in $\tau_{u}$ and increasing in $\tau_{\theta}$.

\subsection{The team solution}

At the team-efficient solution, expected total surplus $\mathbb{E}[\mathrm{TS}]$ is maximized under the constraint that firms use decentralized linear production strategies contingent on endogenous public information (price $p$ or the equivalent variable $z$ ). That is,

$$
\max _{a, b, c} \mathbb{E}[\mathrm{TS}]
$$

subject to $x_{i}=b-a s_{i}+c z$ and $z=u+\beta a \theta$.

19 There are several other cases in which supply has slope $\lambda^{-1}$ and there is no learning from the price: (i) When signals are uninformative about the common parameter $\theta\left(\tau_{\varepsilon}=0\right)$ or when there is no uncertainty ( $\tau_{\theta}=\infty$ and $\theta=\bar{\theta}$ (a.s.)), the price has no information to convey, $a=0$ and $X\left(s_{i}, p\right)=\lambda^{-1}(p-\bar{\theta})$; (ii) When the public statistic is extremely noisy $\left(\tau_{u}=0\right)$ or when $\beta=0$ (in which case there is no payoff externality, either), then public information is pure noise, $a=\lambda^{-1} \tau_{\varepsilon}\left(\tau_{\theta}+\tau_{\varepsilon}\right)^{-1}$, with $X\left(s_{i}, p\right)=\lambda^{-1}\left(p-\mathbb{E}\left[\theta \mid s_{i}\right]\right)$.

20 Downward sloping supply bids have been allowed in some wholesale electricity markets (e.g. in the Nord Pool before 2007). 
Note that in this problem the variable $z$ comes from market clearing and incorporates the conditioning on the price. It is easily seen (see Claim 2 in the Appendix) that the form of the optimal team strategy is the same as the market with the response to private information $a$ as free parameter:

$$
x_{i}=\lambda^{-1}\left(p-\left(\lambda a s_{i}+(1-\lambda a) \mathbb{E}[\theta \mid z]\right)\right) .
$$

As in Section 4.1 this yields a strictly convex WL as a function of $a$ :

$$
\mathrm{WL}(a ; \tau(a))=\frac{1}{2}\left(\frac{(1-\lambda a)^{2}}{\tau(a)(\beta+\lambda)}+\frac{\lambda a^{2}}{\tau_{\varepsilon}}\right) \text { where } \tau(a)=\tau_{\theta}+\tau_{u} \beta^{2} a^{2} \text {. }
$$

Now aggregate inefficiency decreases with increases in $a$ also because price informativeness $\tau(a)$ increases, provided $\tau_{u}>0$, and the average quantity gets close to the full information allocation, and again distributive inefficiency increases with $a$ as dispersion increases. A higher response to private information induces a more informative price (higher $\tau$ ) and reduces aggregate inefficiency but increases distributive inefficiency. The team solution, denoted $a^{\mathrm{T}}$, minimizes WL and optimally trades off the sources of inefficiency among decentralized strategies taking into account that $\tau$ depends on $a$. We characterize next the team solution and the learning externality.

When firms take into account the information content of the price there is a learning externality and an added reason for the market solution to be inefficient. We know from the received literature that the learning externality will tend to make agents put too little weight on private information (Vives 1997, Amador and Weill 2012). The reason is that an agent when responding to its private information does not take into account the improved informativeness of public statistics.

Since WL $(a ; \tau(a))$ is a strictly convex function of $a$, the FOC characterizes the team solution $a^{\mathrm{T}}$

$$
\frac{d \mathrm{WL}}{d a}=\frac{\partial \mathrm{WL}}{\partial a}+\frac{\partial \mathrm{WL}}{\partial \tau} \frac{\partial \tau}{\frac{\partial a}{+}}=0
$$

The solution is unique, and $\lambda^{-1}>a^{T}>0$ provided $\infty>\tau_{\varepsilon}>0$. 
The first term $\partial \mathrm{WL} / \partial a$ corresponds to the direct effect of changing $a$ for a fixed $\tau$ and the second corresponds to the indirect effect through the public precision $\tau$. This second term is the effect of the learning externality and it is negative since $\partial \mathrm{WL} / \partial \tau<0$ and $\partial \tau / \partial a>0$. This implies that for any given $\tau$ we want to increase $a$ from the optimal level with exogenous public information. Indeed, we have that $\partial \mathrm{WL}\left(a_{\mathrm{exo}}^{\mathrm{T}}(\tau) ; \tau\right) / \partial a=0$ and therefore, $\operatorname{dWL}\left(a_{\mathrm{exo}}^{\mathrm{T}}(\tau) ; \tau\right) / d a<0:$ when $a=a_{\mathrm{exo}}^{\mathrm{T}}$, increasing $a$ induces a first order gain making $\tilde{x}$ closer to $x^{o}$ and reducing aggregate inefficiency while there is no first order loss in the trade-off between aggregate and distributive inefficiency. This confirms the idea that the learning externality biases the market solution towards putting too little weight on private information.

We examine now the combined effect of the two (pecuniary and learning) externalities. We know that the learning externality always leads agents to underweight private information and that the pecuniary externality leads to overweight. Depending on the strength of the learning externality we may overcome or not the overweighting result due to the pecuniary externality.

From the FOC $d \mathrm{WL}(a ; \tau(a)) / d a=0$ we obtain that $a^{T}$ fulfills

$$
a=\frac{\tau_{\varepsilon}}{\lambda\left(\tau(a)+\tau_{\varepsilon}\right)+\beta \tau(a)-\Delta(a)},
$$

where $\beta \tau(a)$ corresponds to the pecuniary externality and $\Delta(a)=\frac{(1-\lambda a)^{2} \beta^{2} \tau_{u} \tau_{\varepsilon}}{\lambda \tau(a)} \geq 0$ to the learning externality.

At the market solution, denoted by *, the pecuniary and learning externalities cancel each other exactly when $\beta \tau=\Delta$, in which case $a^{*}=a^{\mathrm{T}}$. This happens when the equilibrium slope of supply is vertical $\left(c^{*} \equiv \partial x_{i} / \partial z=0\right)$. We have that $\beta \tau-\Delta>0$ when $c^{*}>0$ and $\beta \tau-\Delta<0$ when $c^{*}<0$ (see Claim 3 in the Appendix). This suggests that $a^{*}<a^{\mathrm{T}}$ when supply is downward sloping $c^{*}<0$ and $a^{*}>a^{\mathrm{T}}$ when it is upward sloping $c^{*}>0$. The first case happens when $\tau_{u}$ is large, the supply function is downward sloping because the informational component of the 
price prevails, and the learning externality wins over the pecuniary externality. ${ }^{21}$ The second case happens when $\tau_{u}$ is low, the supply function is upward sloping because the allocative effect of the price prevails, and the learning externality is overpowered by the pecuniary externality.

When firms do not respond to the price $\left(c^{*}=0\right)$, the model is equivalent to a quantitysetting model with private information. Indeed, the strategy used by a firm reduces to a Cournot strategy because, in the given parameter constellation, the allocation weight to the price in the supply function $X\left(s_{i}, p\right)=\lambda^{-1}\left(p-\mathbb{E}\left[\theta \mid s_{i}, p\right]\right)$, equal to 1 , exactly matches its informational weight (the weight to the price in $\mathbb{E}\left[\theta \mid s_{i}, p\right]$ ). The result is given in the following proposition.

Proposition 3. Let $\infty>\tau_{\varepsilon}>0$. Then the team problem has a unique solution with $\lambda^{-1}>a^{\mathrm{T}}>0$ and $\operatorname{sgn}\left\{a^{*}-a^{\mathrm{T}}\right\}=\operatorname{sgn}\left\{\beta c^{*}\right\}$.

From the expression for WL we obtain directly that $\left.\frac{d \mathrm{WL}}{d a}\right|_{a=a^{*}}=\lambda a^{*} \sigma_{\varepsilon}^{2} \beta c^{*}$ and $\mathrm{WL}$ is strictly convex with one minimum. The result follows since $a^{*}>0$ when $\tau_{\varepsilon}>0$.

The effect of the two externalities can be seen also, as in Section 4.2, noting that the strategy for firm $i$ is of the form $x_{i}=\lambda^{-1}\left(p-\left(\lambda a s_{i}+(1-\lambda a) \hat{z}\right)\right)$, where $\hat{z}=\mathbb{E}[\theta \mid z], z=\beta a \theta+u$. We have that

$$
\frac{\partial x_{i}}{\partial a}=\left.\frac{\partial x_{i}}{\partial a}\right|_{p, \hat{z}}+\left.\frac{\partial x_{i}}{\partial p} \frac{\partial p}{\partial a}\right|_{\hat{z}}+\frac{\partial x_{i}}{\partial \hat{z}} \frac{\partial \hat{z}}{\partial a},
$$

where the first term corresponds to market behavior, the second to the pecuniary externality with exogenous public information, and the last term to the learning externality:

$$
\frac{\partial x_{i}}{\partial \hat{z}} \frac{\partial \hat{z}}{\partial a}=\left(\frac{\partial x_{i}}{\partial p} \frac{\partial p}{\partial \hat{z}}+\frac{\partial x_{i}}{\partial \hat{z}}\right) \frac{\partial \hat{z}}{\partial a}
$$

21 Recall that the supply function is steeper with higher $\tau_{u}\left(c^{*}\right.$ is decreasing in $\left.\tau_{u}\right)$. 
From Section 4.2 we obtain the effect of the pecuniary externality for given public information at the market solution $a^{*}$ :

$$
\mathbb{E}\left[\left.\left(p-\operatorname{MC}\left(x_{i}\right)\right) \frac{\partial x_{i}}{\partial p} \frac{\partial p}{\partial a}\right|_{\hat{z} \text { ct. }}\right]<0 .
$$

Furthermore, the learning externality has the expected sign

$$
\mathbb{E}\left[\left(p-\operatorname{MC}\left(x_{i}\right)\right)\left(\frac{\partial x_{i}}{\partial \hat{z}} \frac{\partial \hat{z}}{\partial a}\right)\right]>0,
$$

and adding up both externality effects delivers the desired result $\operatorname{sgn}\{\partial \mathbb{E}[\mathrm{TS}] / \partial a\}=\operatorname{sgn}\left\{-\beta c^{*}\right\}$ (see appendix A.2 for more details).

If $\beta=0$ then there is neither a learning nor a pecuniary externality, and the team and market solutions coincide. For $\beta \neq 0, \tau_{\varepsilon}>0$, and $\tau_{u}>0$, the solutions coincide only if $c^{*}=0$. When signals are uninformative $\left(\tau_{\varepsilon}=0\right)$ or perfect $\left(\tau_{\varepsilon}=\infty\right)$ there is no private information, there is no learning externality and the pecuniary externality is internalized at the competitive equilibrium. As a result the team and the market solution coincide (with $a=0$ when $\tau_{\varepsilon}=0$ ). When the price contains no information $\left(\tau_{u}=0\right)$ there is no learning externality and only the pecuniary externality remains with the result that $a^{*}>a^{\mathrm{T}}$ (as in Proposition 1).

In conclusion, in the usual case with upward sloping supply functions, $c^{*}>0$, there is too much dispersion and distributive inefficiency. With downward sloping supply functions, $c^{*}<0$, firms give insufficient weight to private information and there is too much aggregate inefficiency.

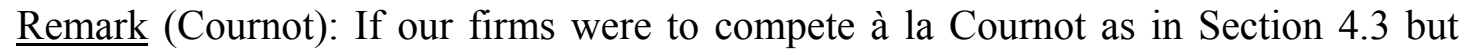
receive an endogenous noisy quantity signal (say the average quantity plus noise) then at the market solution they would rely too little on their private information because of the learning externality. ${ }^{22}$

22 See the online appendix for a precise statement and proof of this result and Bayona (2015). 
Two corollaries follow immediately from Proposition 3. The first is on market quality and the second on the implementation of the team solution with tax-subsidy schemes.

Corollary 3.1 (market quality). At the market solution:

- In relation to the team optimum, when $\beta c^{*}>0$ price informativeness $\tau$ and dispersion $\mathbb{E}\left[\left(x_{i}-\tilde{x}\right)^{2}\right]$ are too high, and aggregate inefficiency $\mathbb{E}\left[\left(\tilde{x}-x^{o}\right)^{2}\right]$ too low. The opposite is true when $\beta c^{*}<0$.

- In relation to the first best (where $\mathbb{E}\left[\left(\tilde{x}-x^{o}\right)^{2}\right]=\mathbb{E}\left[\left(x_{i}-\tilde{x}\right)^{2}\right]=0$ ), price informativeness is too low, and aggregate inefficiency and dispersion are too high.

Corollary 3.2 (implementation of team solution). The team solution $a^{\mathrm{T}}$ can be implemented with a quadratic tax $(\delta / 2) x_{i}^{2}$ on firms where $\delta=\frac{\beta \tau\left(a^{\mathrm{T}}\right)-\Delta\left(a^{\mathrm{T}}\right)}{\tau\left(a^{\mathrm{T}}\right)+\tau_{\varepsilon}}$.

Corollary 3.2 follows since at the team solution

$$
a=\frac{\tau_{\varepsilon}}{\lambda\left(\tau(a)+\tau_{\varepsilon}\right)+\beta \tau(a)-\Delta(a)}=\frac{\tau_{\varepsilon} /\left(\tau(a)+\tau_{\varepsilon}\right)}{\lambda+\delta},
$$

which is the expression for the market responsiveness to private information when the quadratic cost parameter is $\lambda+\delta$. The implementation of the team solution requires, as expected, a tax with $\delta>0$ when $a^{*}>a^{\mathrm{T}}$ (in which case $\beta \tau\left(a^{\mathrm{T}}\right)-\Delta\left(a^{\mathrm{T}}\right)>0$ ) and a subsidy with $\delta<0$ when $a^{*}<a^{\mathrm{T}}$ (in which case $\beta \tau\left(a^{\mathrm{T}}\right)-\Delta\left(a^{\mathrm{T}}\right)<0$ ). The tax or subsidy may be returned/charged in expectation to the firms and therefore it can satisfy budget balance in expected terms. The imposition of an optimal tax $(\delta>0)$ reduces price informativeness (and may increase market depth when $\beta c^{*}>0$ ). ${ }^{23}$

23 See Angeletos and Pavan (2009), Lorenzoni (2010), and Angeletos and La'O (2013) for examples of tax-subsidy schemes to implement team-efficient solutions. Different from them our analysis is based on competition on schedules. 


\subsection{Can more information hurt?}

The question arises as of how the welfare loss WL at the market solution depends on precisions of private and public information as well as on the noise in demand and costs. To elucidate these questions let us consider the model with both an exogenous public signal (adding precision $\kappa^{2} \tau_{\omega}$ ) and the price as endogenous public signal. The effect of the exogenous public signal is the same as adding $\kappa^{2} \tau_{\omega}$ to the prior precision $\tau_{\theta}$ since WL depends only on the total public precision $\tau$ and on private precision $\tau_{\varepsilon} .{ }^{24}$ Therefore the comparative statics of $\tau_{\theta}$ and $\tau_{\omega}$ will be identical.

We know that WL is a strictly convex function of $a$ attaining a minimum at the team-efficient solution $a^{\mathrm{T}}$. It is immediate then that $\operatorname{WL}\left(a^{\mathrm{T}}\right)$ is decreasing in $\tau_{\varepsilon}, \tau_{u}$ and $\tau_{\theta}$. This is so since $\mathrm{WL}$ is decreasing in $\tau_{\varepsilon}, \tau_{u}$ and $\tau_{\theta}$ for a given $a$ and $d \mathrm{WL}\left(a^{\mathrm{T}}\right) / d a=0$. Things are potentially different at the market solution $a^{*}$ since then $d \mathrm{WL}\left(a^{*}\right) / d a$ is positive or negative depending on whether $a^{*}>a^{\mathrm{T}}$ or $a^{*}<a^{\mathrm{T}}$. Since $a^{*}$ is decreasing in $\tau_{u}$ and $\tau_{\theta}$, and increasing in $\tau_{\varepsilon}$ (see Proposition 2) we have thus that $\operatorname{WL}\left(a^{*}\right)$ is decreasing in $\tau_{u}$ and $\tau_{\theta}$ when $a^{*}>a^{\mathrm{T}}$ and in $\tau_{\varepsilon}$ when $a^{*}<a^{\mathrm{T}} .{ }^{25}$ It would be possible in principle that increasing precisions $\tau_{u}$ and $\tau_{\theta}$ increases the welfare loss when $a^{*}<a^{\mathrm{T}}$ when the direct effect of the increase of $\tau_{u}$ or $\tau_{\theta}$ is dominated by the indirect effect via the induced decrease in $a^{*}$ (and similarly for an increase in $\tau_{\varepsilon}$ when $\left.a^{*}>a^{\mathrm{T}}\right)$. We can check, however, that $\mathrm{WL}\left(a^{*}\right)$ is always decreasing in $\tau_{\theta}$ and $\tau_{u}$. This need not be the case when changing $\tau_{\varepsilon}$. In any case, as the information precisions $\tau_{\theta}, \tau_{u}$ and $\tau_{\varepsilon}$ tend to infinity $\operatorname{WL}\left(a^{*}\right)$ tends to $0 .{ }^{26}$ The following proposition summarizes the results.

24 Note, however, that changes in $\tau_{\theta}$ will change ETS $^{\circ}$.

25 We have that $a^{T}$ is increasing in $\tau_{\varepsilon}$ and decreasing in $\tau_{\theta}$ (since $\partial^{2} \mathrm{WL} / \partial a \partial \tau_{\varepsilon}<0$, $\partial^{2} \mathrm{WL} / \partial a \partial \tau_{\theta}>0$, and WL is strictly convex in $\left.a\right)$; and with simulations we obtain that $a^{T}$ is hump-shaped or decreasing in $\tau_{u}$.

26 This follows since as $\tau_{\varepsilon} \rightarrow \infty, a^{*} \rightarrow \lambda^{-1}$; and as $\tau_{\theta}$ or $\tau_{u} \rightarrow \infty, a^{*} \rightarrow 0$ and $\tau \rightarrow \infty$. 
Proposition 4. The welfare loss at the team-efficient solution is decreasing in $\tau_{\varepsilon}, \tau_{\omega}, \tau_{u}$ and $\tau_{\theta}$. The welfare loss at the market solution is also decreasing in $\tau_{\omega}, \tau_{\theta}$ and $\tau_{u}$, and it may be decreasing or increasing in $\tau_{\varepsilon}$ (it will be increasing for $\beta>\lambda$ and $\tau_{\varepsilon} / \tau_{\theta}$ small enough). As any of the precisions $\tau_{\theta}, \tau_{u}, \tau_{\omega}$ and $\tau_{\varepsilon}$ tend to infinity welfare losses tend to zero.

More precise public $\left(\tau_{\omega}\right)$ or private $\left(\tau_{\varepsilon}\right)$ information reduces the welfare loss at the team-efficient solution. This is in accordance with the results in Angeletos and Pavan (2007, 2009) where more information can not hurt when it is used efficiently. The welfare loss at the market solution is also always decreasing with the precision of public information. However, the welfare loss at the market solution may be increasing with the precision of private information when the market calls already for a too large response to private information. The reason is that an increase in the precision of private information will increase the response of an agent to his private signal and this indirect effect may dominate.

The welfare result of the market solution is in contrast with received results in the literature where more public information may be damaging to welfare (Burguet and Vives 2000; Morris and Shin 2002; Angeletos and Pavan 2007; Amador and Weill 2010, 2012). In those papers more public information discourages the use and/or acquisition of private information. In the present paper this also happens but the direct effect of public information provision prevails. ${ }^{27}$

\subsection{Application: Business cycle policy}

Consider a standard "island" economy business cycle model with CRR utilities and CES aggregators augmented with incomplete information (see Angeletos et al. 2015 and Colombo et al. (2014) for models of the same family). A reduced form of the model has players being the islands in the economy (with representative household

27 A possible extension of the model would study the private incentives to acquire information (as in Vives 1988; Burguet and Vives 2000; Hellwig and Veldkamp 2009; Myatt and Wallace 2012; Llosa and Venkateswaran 2013; and Colombo et al. 2014). 
and firm), the actions are productions (which can be strategic substitutes or strategic complements), and types the local information sets consisting of exogenous and endogenous private and public signals (the endogenous public signal being a noisy aggregate quantity, say a macro forecast). The equilibrium is in log-linear strategies (of the Bayesian-Cournot type) and it is unique under certain parametric conditions. The team welfare function is analogous to ours but in constant elasticity form and the optimum is found in the class of log-linear decentralized strategies. The model so far is akin to the Cournot version of our model (see Section 4.3), albeit quite a bit more complex. The result is that if prices are flexible and there are no endogenous signals the equilibrium is team efficient (as in our Cournot economy). ${ }^{28}$ With endogenous signals there is an informational externality and the equilibrium is not team efficient. In this case the optimal policy is countercyclical in the sense that it induces agents to put more weight on their private information to internalize the informational externality. This is, indeed, the result we obtain in our Cournot version of the model with an endogenous public signal (see the online appendix). However, we may ask where does the aggregate public signal comes from. If the signal is an average price across islands, something very plausible, then the results of the present paper apply: a) Even if prices are flexible and agents are naïve and do not learn from prices the equilibrium is not team efficient; b) In the case with strategic substitutes competition, agents put too much weight on their private information and optimal policy should be pro-cyclical. We see, therefore, how providing a plausible interpretation for the public signal as a price index we overturn optimal policy.

\section{The case of (exogenous) upward sloping demand}

We consider in this section the case of $\beta<0$ (i.e. demand is upward sloping). The situation may arise, for example, in the case of a good which is addictive. Assume that $\beta+\lambda>0$ to insure that $\mathrm{TS}$ is strictly concave at symmetric solutions. The characterization of equilibrium with naïve (Section 4.1) or sophisticated firms taking into account the information in the price (Proposition 2 in Section 5.1) holds. In the latter case a high price is good news (entailing lower costs) and supply functions are

28 This result will not hold in general in price-setting economies with complementarities (see, e.g., Hellwig 2005 and Lorenzoni 2010). 
flatter with asymmetric information. ${ }^{29}$ When $\beta<0$ a high price conveys the good news that average quantity tends to be high and that costs therefore tend to be low. In this case, increasing $\tau_{u}$ induces a smaller slope of supply.

With respect to welfare, the expression in Proposition 1 also holds and the general result is that $\operatorname{sgn}\left\{a_{\text {exo }}^{*}(\tau)-a_{\text {exo }}^{\mathrm{T}}(\tau)\right\}=\operatorname{sgn}\{\beta\}$. As a consequence, the sign of the pecuniary externality depends on whether $\beta$ is positive (downward sloping demand) or negative (upward sloping demand). In the latter case a higher output leads to a higher price. Indeed, consistently with the result in Proposition 1 it follows that at the market solution for given $\tau$ the sign of the pecuniary externality depends on the sign of $\beta, \operatorname{sgn}\{\partial \mathbb{E}[\mathrm{TS}] / \partial a\}=\operatorname{sgn}\{-\beta\}$.

With endogenous public information we know that the learning externality always leads agents to underweight private information, and the pecuniary externality leads also to underweight when demand is upward sloping. It follows that for $\beta<0$ we will have always underweighting of private information, and $a^{*}<a^{\mathrm{T}}$. Proposition 3 and its corollaries hold. Proposition 4 also holds whenever $2 \beta+\lambda>0.30$

\section{Demand schedule competition and optimal transaction taxes}

In this section we reinterpret the model in terms of competition in demand schedules. Let a buyer of a homogenous good with unknown ex post value $\theta$ face an inverse supply $p=\alpha+u+\beta \tilde{y}$, where $\tilde{y}=\int_{0}^{1} y_{i} d i$ and $y_{i}$ is the demand of buyer $i$. The suppliers face a cost of supply of $\left(\alpha+u+\beta \frac{\tilde{y}}{2}\right) \tilde{y}$. The marginal cost of supply is increasing (decreasing) in the amount supplied when $\beta>0(\beta<0)$. The case $\beta<0$ may correspond, for example, to a situation where there is learning by doing in the supply. The buyer's net benefit is given by $\pi_{i}=(\theta-p) y_{i}-(\lambda / 2) y_{i}^{2}$, where $(\lambda / 2) y_{i}^{2}$ is a transaction or opportunity cost (or an adjustment for risk aversion). The timing of events runs parallel to one in the basic model in Section 2 letting $y_{i} \equiv-x_{i}$. We

29 This follows because we assume that $\beta+\lambda>0$.

30 This regularity condition is assumed in Section 4. 
illustrate the results for a financial market (another illustration would have firms hiring labor of unknown productivity).

\section{Traders in a financial market. ${ }^{31}$}

Informed speculators have information on the liquidation value $\theta$ of a risky asset and face quadratic transaction costs (alternatively, the parameter $\lambda$ proxies for risk aversion). Liquidity suppliers trade according to the elastic aggregate demand $(\alpha+u-p) / \beta$, where $u$ is random. When $\beta>0$ liquidity suppliers buy (sell) when the price is low (high); when $\beta<0$, liquidity suppliers buy (sell) when the price is high (low). In this latter case we could interpret liquidity suppliers as program traders following a portfolio insurance strategy. ${ }^{32}$

Our results apply. With $\beta>0$ and downward-sloping demand schedules for informed traders, there is overreaction to private information. This will happen when the volume of liquidity trading is high (i.e. when $\tau_{u}$ is low). In this case a Tobin-style tax on privately informed speculators is warranted. If the tax is set at the optimal level (see Corollary 3.2) it will reduce the responsiveness to private information of speculators and implement the team optimum. The tax may increase market depth. It is worth noting again that the tax is not optimal because of a Hirshleifer effect of prices being "too" informative and destroying insurance opportunities. ${ }^{33}$ The tax corrects a pecuniary externality which arises because informed traders condition on prices when trading.

To levy a tax only on privately informed speculators may not be feasible. In fact, a common criticism to the Tobin tax is that it cannot distinguish between speculators and liquidity suppliers. It is easy to see, however, that an appropriate tax on all traders will also work. This is so since the responsiveness to information of informed

31 A variation of this example can be used to model Treasury or liquidity auctions.

32 See Gennotte and Leland (1990). Hendershott and Seasholes (2009) find that program trading accounts for almost $14 \%$ of the average daily market volume at the NYSE in 1999-2005 and that program traders lose money on average.

33 Dow and Rahi (2000) consider quadratic transaction taxes in models with risk averse informed traders and find conditions under which a transaction tax can be Pareto improving even if the tax revenue is wasted. Subrahmanyam (1998) also considers quadratic transaction taxes and finds that the tax reduces market liquidity. 
speculators decreases not only with their transaction cost $\lambda$ but also with $|\beta|$ (see Proposition 2). Consider thus a quadratic tax $\delta$ on both informed, and liquidity traders. Then the inverse supply is given by $p=\alpha+u+(\beta+\delta) \tilde{y}$ and at the market solution $a^{*}(\delta)$,

$$
a=\frac{\tau_{\varepsilon}}{(\lambda+\delta)\left(\tau_{\varepsilon}+\tau_{\theta}+(\beta+\delta)^{2} \tau_{u} a^{2}\right)}
$$

Consider the case with $\beta>0$ and downward-sloping demand schedules for informed traders $\left(c^{*}>0\right)$. We know then that $a^{*}>a^{\mathrm{T}}>0$ when $\delta=0$ (Proposition 3$)$. It is immediate that $a^{*}(\delta)$ decreases with $\delta$, and ranges from $a^{*}$ to 0 as $\delta$ goes from 0 to $\infty$. Therefore, there is a $\delta>0$ for which $a^{*}(\delta)=a^{T}$. This is the common transaction tax that implements the team solution. It is worth noting that this $\delta$ is strictly lower than the transaction tax targeted only to speculators (as given in Corollary 3.2). In the parameter region where demand schedules for the informed are upward sloping, those traders underreact to their private information. The same applies in the case $\beta<0$. In those cases a transaction subsidy would be optimal.

\section{Internal welfare benchmark}

In this section we explore a different welfare benchmark where only the welfare of the producers (firms) is taken into account. This is a collusive benchmark where the welfare of consumers is disregarded. We term it the internal team solution and consider directly the case where firms do take into account the information content of prices. We allow both positive and negative $\beta$ and assume that $2 \beta+\lambda>0$, which guarantees that profits are strictly concave in output at symmetric solutions $\left(\partial^{2} \pi /(\partial x)^{2}=-(2 \beta+\lambda)<0\right)$.

At the internal team-efficient solution, expected average profit $\mathbb{E}[\tilde{\pi}]$,where $\tilde{\pi}=\int_{0}^{1} \pi_{i} d i$, is maximized under the constraint that firms use decentralized linear strategies. Since the solution is symmetric we have that $\mathbb{E}[\tilde{\pi}]=\mathbb{E}\left[\pi_{i}\right]$. This is the cooperative solution from the firms' perspective. That is, 


$$
\max _{a, b, c} \mathbb{E}\left[\pi_{i}\right]
$$

subject to $x_{i}=b-a s_{i}+c z$ and $z=u+\beta a \theta$.

Note again that the team strategy conditions on the variable $z=u+\beta a \theta$ which is informationally equivalent to the price. It should be clear that the market solution, not even with complete information, will attain the full information cooperative outcome (denoted $\mathrm{M}$ for monopoly, for which $\left.x^{\mathrm{M}}=(\lambda+2 \beta)^{-1}(\alpha+u-\theta)\right)$ where joint profits are maximized under full information. This is so since the market solution does not internalize the competition (payoff) externality and therefore if $\beta \neq 0$ it will produce an expected output $\mathbb{E}\left[\tilde{x}^{*}\right]=\alpha(\beta+\lambda)^{-1}$ which is too high (low) with $\beta>0$ $(\beta<0)$ in relation to the optimal $\mathbb{E}\left[x^{\mathrm{M}}\right]=\alpha(2 \beta+\lambda)^{-1}$. Furthermore, the market solution does not internalize the externalities in the use of information arising from price-contingent strategies. At the internal team (IT) benchmark, joint profits are maximized and information-related externalities internalized with decentralized strategies. ${ }^{34}$ The question is whether the market solution allocates the correct weights (from the firms' collective welfare viewpoint) to private and public information. We show that the answer to this question is qualitatively similar to the one derived when analyzing the total surplus team benchmark but in this case with a larger bias towards the market displaying too much weight on private information.

As before, it can be seen that the internal team-efficient solution minimizes, over the restricted strategies, the expected loss $\Omega$ with respect to the full information cooperative outcome $x^{\mathrm{M}}$, and that

$$
\Omega=\left((2 \beta+\lambda) \mathbb{E}\left[\left(\tilde{x}-x^{\mathrm{M}}\right)^{2}\right]+\lambda \mathbb{E}\left[\left(x_{i}-\tilde{x}\right)^{2}\right]\right) / 2 .
$$

The first term in the sum corresponds to aggregate inefficiency in the average quantity, which is proportional to $\mathbb{E}\left[\left(\tilde{x}-x^{\mathrm{M}}\right)^{2}\right]$, and the second term to productive inefficiency, which is proportional to $\mathbb{E}\left[\left(x_{i}-\tilde{x}\right)^{2}\right]$.

34 Indeed, when $\beta=0$ there are no externalities (payoff or informational) and the internal team and market solutions coincide. 
It can be checked that the form of the internal optimal team strategy is $x_{i}=(\lambda+\beta)^{-1}\left(p-\left(\gamma s_{i}+(1-\gamma) \mathbb{E}[\theta \mid z]\right)\right)$ where $\gamma=(\lambda+\beta) a$ (while at the market solution we have that $\gamma=\lambda a$ ). The loss at any candidate internal team solution (which internalizes the competition payoff externality and for which $\left.\mathbb{E}[\tilde{x}]=\alpha(2 \beta+\lambda)^{-1}\right)$ will depend only on the response to private information $a$ since at this candidate solution we have $\mathbb{E}\left[\left(\tilde{x}-x^{\mathrm{M}}\right)^{2}\right]=(1-(\lambda+\beta) a)^{2} /\left(\tau(2 \beta+\lambda)^{2}\right)$ and $\mathbb{E}\left[\left(x_{i}-\tilde{x}\right)^{2}\right]=a^{2} / \tau_{\varepsilon}$. This yields a strictly convex $\Omega$ as a function of $a$. As before, changing $a$ has opposite effects on both sources of the loss. Now the internal team solution optimally trades off the sources of the loss with respect to the responsiveness to private information among decentralized strategies which internalize the competition payoff externality.

In this case at the market solution there is as before a combined, pecuniary and learning, price-contingent strategy externality $(\mathrm{PE}+\mathrm{LE})$ in the use of private information, and also a competition payoff (CE) externality through the impact of aggregate output on price in the use of information, since even with full information the market solution is not efficient (i.e. cooperative). The impact of the externalities on the response to private information can be assessed similarly as before. The market takes the public statistic $z$ or $p$ as given while the internal team solution takes into account all externalities:

$$
\begin{aligned}
\frac{\partial \mathbb{E}\left[\pi_{i}\right]}{\partial a}= & \underbrace{\mathbb{E}\left[\left(p-M C\left(x_{i}\right)\right)\left(\frac{\partial x_{i}}{\partial a}\right)_{z}\right]_{\text {Market }}}+ \\
& \mathbb{E} \underbrace{[\underbrace{}_{\mathrm{PE}+\mathrm{LE}}}+ \\
& \underbrace{\left[x_{i}\left(\frac{\partial p}{\partial \tilde{x}} \frac{\partial \tilde{x}}{\partial a}\right)\right]}]_{\mathrm{CE}} .
\end{aligned}
$$

The market term is null at the market solution and the sum of the PE+LE and CE terms at the market solution can be evaluated as follows: 


$$
\left.\frac{\partial \mathbb{E}\left[\pi_{i}\right]}{\partial a}\right|_{a=a^{*}}=-\beta a^{*}\left(c^{*} \lambda \sigma_{\varepsilon}^{2}+\left(c^{*} \beta-1\right)^{2} \sigma_{\theta}^{2}\right) .
$$

It is worth noting that while, as before, $\operatorname{sgn}\{\mathrm{PE}+\mathrm{LE}\}=\operatorname{sgn}\left\{-\beta c^{*}\right\}$ we have that $\operatorname{sgn}\{\mathrm{CE}\}=\operatorname{sgn}\{-\beta\}$ since $\left(c^{*} \beta-1\right)^{2} \sigma_{\theta}^{2}>0$, and therefore the CE term will call for a lower (higher) response to private information with downward (upward) sloping demand than the market solution. If $\beta>0$ a high price indicates high costs. If, say, costs are high $(\theta-\bar{\theta}>0)$ then an increase in $a$ will increase $p$ $\left(\frac{\partial p}{\partial \tilde{x}} \frac{\partial \tilde{x}}{\partial a}=-\beta(c \beta-1)(\theta-\bar{\theta})>0\right.$ since at the market solution $\left.c \beta<1\right)$ while $x_{i}$ will tend to be low (since at the market solution $\left.\mathbb{E}\left[(\theta-\bar{\theta}) x_{i}\right]=a \sigma_{\theta}^{2}(c \beta-1)<0\right)$. This means that if $\beta>0, \mathrm{CE}<0$ and $a$ must be reduced. Similarly, we have that $\mathrm{CE}>0$ if $\beta<0$. The results on the payoff externality $\mathrm{CE}$ are in line with the results obtained by Angeletos and Pavan (Section 6.5, 2007) with non price-contingent strategies. We will see how the effect of the PE+LE term may overturn this result when $c<0$.

The next proposition characterizes the response to private information.

Proposition 5. Let $\infty>\tau_{\varepsilon}>0$. Then the internal team problem has a unique solution with $(\lambda+\beta)^{-1}>a^{\mathrm{IT}}>0$, and $\operatorname{sgn}\left\{a^{*}-a^{\mathrm{IT}}\right\}=\operatorname{sgn}\left\{\beta\left(c^{*} \lambda \sigma_{\varepsilon}^{2}+\left(c^{*} \beta-1\right)^{2} \sigma_{\theta}^{2}\right)\right\}$.

If $c^{*} \geq 0$ then $\operatorname{sgn}\left\{a^{*}-a^{\mathrm{IT}}\right\}=\operatorname{sgn}\{\beta\}$. Therefore, as before, under $\beta<0$, there is too little response to private information, $a^{*}<a^{\mathrm{IT}}$. Indeed, the characterization yields the same qualitative result as in the previous section if $c^{*}>0$ : too much or too little response to private information depending on the sign of $\beta$. In this case, however, if agents use Cournot strategies (i.e., if $c^{*}=0$ ) then the market is not internal teamefficient. This should not be surprising when one considers that, when $c^{*}=0$, the combined externality for the use of price-contingent strategies is nil, yet the competition payoff externality is not internalized, as firms set a quantity that is too large (small) when $\beta>0(\beta<0)$. If $\beta>0$ and $c^{*}<0$, then $c^{*} \lambda \sigma_{\varepsilon}^{2}+\left(c^{*} \beta-1\right)^{2} \sigma_{\theta}^{2}>0$ 
for $c^{*}$ close to zero or sufficiently negative $\left(\tau_{u}\right.$ large). Only for intermediate values of $c^{*}$ we have $c^{*} \lambda \sigma_{\varepsilon}^{2}+\left(c^{*} \beta-1\right)^{2} \sigma_{\theta}^{2}<0$ and $a^{\mathrm{TT}}>a^{*}$. With $\beta>0$ the market will bias the solution more towards putting too high a weight on private information since we may have $c^{*} \lambda \sigma_{\varepsilon}^{2}+\left(c^{*} \beta-1\right)^{2} \sigma_{\theta}^{2}>0$ even if $c^{*}<0$.

Application. Monopolistic competition. The model applies also to a monopolistically competitive market with quantity-setting firms; in this case, either $\beta>0$ (goods are substitutes) or $\beta<0$ (goods are complements). Firm $i$ faces the inverse demand for its product, $p_{i}=\alpha+u-\beta \tilde{x}-(\lambda / 2) x_{i}$, and has costs $\theta x_{i}$. Each firm uses a supply function that is contingent on its own price: $X\left(s_{i}, p_{i}\right)$ for firm $i$. It follows then that observing the price $p_{i}$ is informationally equivalent (for firm $i$ ) to observing $p \equiv \alpha+u-\beta \tilde{x}$. Under monopolistic competition, the total surplus function (consistent with the differentiated demand system) is slightly different:

$$
\mathrm{TS}=(\alpha+u-\theta) \tilde{x}-\left(\beta \tilde{x}^{2}+(\lambda / 2) \int_{0}^{1} x_{i}^{2} d i\right) / 2 .
$$

Here the market is not efficient under complete information because price is not equal to marginal cost. Each firm has some residual market power. The welfare results of Section 5 do not apply but those of the present section apply when firms collude. It is interesting to note then that, if agents cannot use price-contingent strategies (as in the cases of Cournot or Bertrand competition), Angeletos and Pavan (Section 6.5, 2007) argue that with strategic substitutability ( $\beta>0$ in our case) we would have always excessive response to private information in contrast with the case with supply functions as strategies, where either excessive or insufficient response to private information is possible.

\section{Concluding remarks.}

We find that price-contingent strategies, on top of the usual learning externality, introduce a pecuniary externality in the use of private information which induces agents to overweight private information (with decreasing marginal utility). This externality dominates the usual learning-from-prices externality when the allocative role of prices prevails over their informational role. The inefficiency of the market 
solution opens the door to the possibility that more precise public or private information will lead to an increased welfare loss. This is the case when the market already calls for a too large response to private information, then more precise private information exacerbates the problem (but not more precise public information).

The practical implication of the result is that in market games, where agents condition on prices, the presumption that agents rely too much on public information and too little in private information will not hold. Efficiency can be restored with an optimal tax, which in the case of financial markets is a quadratic Tobin-like tax, and which induces traders to internalize the externalities they generate. The results have also implications for business cycle policy when firms have private information on productivity. They may rationalize the use of pro-cyclical policy to moderate the response of firms to their private information. The policy implications have to be understood as illustrations of the results in the context of the very stylized model presented.

The results extend to an economy which is not efficient with full information. Then the potential bias towards putting too much weight on private information is increased. It follows that received results on the optimal relative weights to be placed on private and public information (when the latter is exogenous) may be overturned when the informational role of the price conflicts with its allocative role and the former is important enough.

\section{$\underline{\text { Appendix }}$}

\section{$\underline{\text { Equilibrium and welfare characterization results: proofs }}$}

In this appendix we allow for both positive and negative $\beta$ and assume $\beta+\lambda>0$.

Given that

$$
\mathrm{TS} \equiv\left(\alpha+u-\beta \frac{\tilde{x}}{2}\right) \tilde{x}-\int_{0}^{1}\left(\theta x_{i}+\frac{\lambda}{2} x_{i}^{2}\right) d i
$$

the first-best (full information) allocation has all firms producing the same amount, $x^{o}$. (Since $\beta+\lambda>0$, TS is strictly concave for symmetric solutions.) Using the fact that $x^{o}(\lambda+\beta)=\alpha+u-\theta \quad, \quad$ we obtain $\quad \operatorname{TS}^{o}=(\lambda+\beta)\left(x^{o}\right)^{2} / 2 \quad$ and 
$\mathrm{TS}^{o}-\mathrm{TS}=\left((\beta+\lambda)\left(\tilde{x}-x^{o}\right)^{2}+\lambda \int_{0}^{1}\left(x_{i}-\tilde{x}\right)^{2} d i\right) / 2$ for a symmetric allocation. This yields

$$
\mathrm{WL}=\left((\beta+\lambda) \mathbb{E}\left[\left(\tilde{x}-x^{o}\right)^{2}\right]+\lambda \mathbb{E}\left[\left(x_{i}-\tilde{x}\right)^{2}\right]\right) / 2 .
$$

We consider in turn the cases of exogenous and endogenous public information.

\section{$\underline{\text { A.1 Exogenous public information }}$}

Claim 1: The strategy at the team solution with exogenous public information is of the same form as the market solution: $X\left(s_{i}, z ; p\right)=\lambda^{-1}\left(p-\left(\gamma s_{i}+(1-\gamma) \hat{z}\right)\right)$ where $\hat{z} \equiv \mathbb{E}[\theta \mid z]$

Proof: The market strategy with exogenous public information is $X\left(s_{i}, z ; p\right)=\lambda^{-1}\left(p-\left(\gamma s_{i}+(1-\gamma) \hat{z}\right)\right)$ where $\gamma=\tau_{\varepsilon}\left(\tau_{\varepsilon}+\tau\right)^{-1}, \tau=\tau_{\theta}+\tau_{\omega} \kappa^{2}$. The team solution with exogenous public information $a_{e x o}^{*}(\tau)$ solves program $\left(\mathbf{T}_{\text {exo }}\right)$.

It can be checked that $\partial^{2} \mathbb{E}[\mathrm{TS}] / \partial^{2} \hat{b}<0$ whenever $\beta+\lambda>0, \partial^{2} \mathbb{E}[\mathrm{TS}] / \partial^{2} c<0$, and $\partial^{2} \mathbb{E}[\mathrm{TS}] / \partial^{2} \hat{c}<0$ whenever $\operatorname{var}[p]>0$. Given that $\partial x_{i} / \partial \hat{b}=1, \partial x_{i} / \partial c=-z$, and $\partial x_{i} / \partial \hat{c}=p$ we can optimize $\mathbb{E}[\mathrm{TS}]$ with respect to $b, c, \hat{c}$ to obtain

$$
\begin{aligned}
& \frac{\partial \mathbb{E}[\mathrm{TS}]}{\partial \hat{b}}=\mathbb{E}\left[\left(p-\operatorname{MC}\left(x_{i}\right)\right)\right]=0, \\
& \frac{\partial \mathbb{E}[\mathrm{TS}]}{\partial c}=\mathbb{E}\left[\left(p-\operatorname{MC}\left(x_{i}\right)\right) z\right]=0, \\
& \frac{\partial \mathbb{E}[\mathrm{TS}]}{\partial \hat{c}}=\mathbb{E}\left[\left(p-\operatorname{MC}\left(x_{i}\right)\right) p\right]=0,
\end{aligned}
$$

where $\operatorname{MC}\left(x_{i}\right)=\theta+\lambda x_{i}$. The constraint $\mathbb{E}\left[p-\operatorname{MC}\left(x_{i}\right)\right]=0$ can be seen equivalent to $(1-\lambda \hat{c}) \mathbb{E}[p]=\lambda \hat{b} \quad$ (using the fact that $\mathbb{E}[\theta]=\mathbb{E}\left[s_{i}\right]=\mathbb{E}[z]=0 \quad$ ), and $\mathbb{E}\left[\left(p-\operatorname{MC}\left(x_{i}\right)\right) p\right]=0$ equivalent to $(1-\lambda \hat{c}) \mathbb{E}\left[p^{2}\right]=\lambda \hat{b} \mathbb{E}[p]$ (using the assumption that $\mathbb{E}[\theta p]=0$ and therefore $\mathbb{E}[z p]=0$ ). The equations $(1-\lambda \hat{c}) \mathbb{E}[p]=\lambda \hat{b}$ and $(1-\lambda \hat{c}) \mathbb{E}\left[p^{2}\right]=\lambda \hat{b} \mathbb{E}[p]$ can hold with $\mathbb{E}[p]>0$ and $\operatorname{var}[p]>0$ if and only if $1-\lambda \hat{c}=\lambda \hat{b}=0$. (In equilibrium we necessarily have 
$\mathbb{E}[p]>0$, provided $\alpha>0$, and $\operatorname{var}[p]>0$ provided $\sigma_{\theta}^{2}>0$.) Therefore we conclude that $\hat{c}=\lambda^{-1}$ and $\hat{b}=0$. Furthermore, $\mathbb{E}\left[\left(p-\operatorname{MC}\left(x_{i}\right)\right) z\right]=0$ can be seen equivalent to $c=\frac{(1-a \lambda) k \tau_{w}}{\lambda\left(\tau_{\theta}+\tau_{w} k^{2}\right)}$ (using the fact that $\left.\mathbb{E}[z p]=0\right)$. Note that $c z=\lambda^{-1}(1-\lambda a) \hat{z}$ since $\hat{z} \equiv \mathbb{E}[\theta \mid z]=\frac{k \tau_{w}}{\tau_{\theta}+\tau_{w} k^{2}} z$. It follows therefore that we can write the team strategy as $x_{i}=-a s_{i}-\lambda^{-1}(1-\lambda a) \hat{z}+\lambda^{-1} p=\lambda^{-1}\left(p-\left(\gamma s_{i}+(1-\gamma) \hat{z}\right)\right)$ where $\gamma=\lambda a$.

It follows that the welfare loss at any candidate team solution will depend only on the response to private information $\gamma$, or equivalently on the response to private information in the strategy of a firm: $a=\lambda^{-1} \gamma$. We have then that $\tilde{x}=(\alpha+u-(\lambda a \theta+(1-\lambda a) \hat{z})) /(\beta+\lambda)$ and, using the expression for $x^{o}$, we find that $\tilde{x}-x^{o}=(1-\lambda a)(\theta-\hat{z}) /(\beta+\lambda) \quad$. Since $\quad \tau=(\operatorname{var}[\theta \mid z])^{-1} \quad$ we obtain $\mathbb{E}\left[\left(\tilde{x}-x^{o}\right)^{2}\right]=(1-\lambda a)^{2} /\left(\tau(\beta+\lambda)^{2}\right) \quad$. Similarly $\quad$ we obtain $x_{i}-\tilde{x}=-\lambda^{-1} \gamma\left(s_{i}-\theta\right)=-a \varepsilon_{i}$ and, conclude that $\mathbb{E}\left[\left(x_{i}-\tilde{x}\right)^{2}\right]=a^{2} \sigma_{\varepsilon}^{2}$. It follows from the expression of the welfare loss WL that

$$
\mathrm{WL}(a ; \tau)=\frac{1}{2}\left(\frac{(1-\lambda a)^{2}}{\tau(\beta+\lambda)}+\frac{\lambda a^{2}}{\tau_{\varepsilon}}\right) .
$$

The team solution minimizes $\operatorname{WL}(a ; \tau)$ over $a$, yielding $a_{\mathrm{exo}}^{\mathrm{T}}(\tau)=\frac{\tau_{\varepsilon}}{\lambda\left(\tau_{\varepsilon}+\tau\right)+\beta \tau}$. Proposition 1 follows and for $\infty>\tau_{\varepsilon}>0$ and $\sigma_{u}^{2} \geq 0$, $\operatorname{sgn}\left\{a_{\text {exo }}^{*}(\tau)-a_{\text {exo }}^{\mathrm{T}}(\tau)\right\}=\operatorname{sgn}\{\beta\}$.

Lemma: At the market solution for given public precision $\tau$ we have that

$$
\left.\frac{\partial \mathbb{E}[\mathrm{TS}]}{\partial a}\right|_{a=a_{\text {exo }}^{*}}=\mathbb{E}\left[\left.\left(p-\operatorname{MC}\left(x_{i}\right)\right) \frac{\partial x_{i}}{\partial p} \frac{\partial p}{\partial a}\right|_{a=a_{e \times 0}^{*}}\right]=-\beta \lambda(\beta+\lambda)^{-1} a_{e x o}^{*} \sigma_{\varepsilon}^{2} .
$$

\section{Proof:}

We derive first the expression for $\partial \mathbb{E}[\mathrm{TS}] / \partial a$. We can write total surplus as follows: 


$$
\mathrm{TS}=\int_{0}^{1} \varphi\left(x_{i}, \tilde{x}\right) d i
$$

where $\varphi\left(x_{i}, \tilde{x}\right)=(\alpha+u-\beta \tilde{x}) x_{i}-C\left(x_{i}\right)+(\beta / 2) \tilde{x}^{2}$. We have that

$$
\frac{\partial \mathbb{E}[\mathrm{TS}]}{\partial a}=\mathbb{E}\left[\int_{0}^{1}\left(\frac{\partial \varphi}{\partial x_{i}} \frac{\partial x_{i}}{\partial a}+\frac{\partial \varphi}{\partial \tilde{x}} \frac{\partial \tilde{x}}{\partial a}\right) d i\right]=\mathbb{E}\left[\int_{0}^{1}\left(\frac{\partial \varphi}{\partial x_{i}} \frac{\partial x_{i}}{\partial a}\right) d i\right]
$$

since $\int_{0}^{1}\left(\frac{\partial \varphi}{\partial \tilde{x}} \frac{\partial \tilde{x}}{\partial a}\right) d i=\frac{\partial \tilde{x}}{\partial a}\left(\int_{0}^{1}\left(\beta\left(\tilde{x}-x_{i}\right)\right) d i\right)=0$ and $\tilde{x}=\int_{0}^{1} x_{i} d i$. Furthermore, $\partial \varphi / \partial x_{i}=p-\operatorname{MC}\left(x_{i}\right)$. Therefore, because of symmetry,

$$
\frac{\partial \mathbb{E}[\mathrm{TS}]}{\partial a}=\mathbb{E}\left[\int_{0}^{1}\left(\frac{\partial \varphi}{\partial x_{i}} \frac{\partial x_{i}}{\partial a}\right) d i\right]=\int_{0}^{1} \mathbb{E}\left[\frac{\partial \varphi}{\partial x_{i}} \frac{\partial x_{i}}{\partial a}\right] d i=\mathbb{E}\left[\frac{\partial \varphi}{\partial x_{i}} \frac{\partial x_{i}}{\partial a}\right] \text { and }
$$

from $\frac{\partial x_{i}}{\partial a}=\left.\frac{\partial x_{i}}{\partial a}\right|_{p}+\frac{\partial x_{i}}{\partial p} \frac{\partial p}{\partial a}$, we obtain

$$
\frac{\partial \mathbb{E}[\mathrm{TS}]}{\partial a}=\mathbb{E}\left[\left.\left(p-\operatorname{MC}\left(x_{i}\right)\right) \frac{\partial x_{i}}{\partial a}\right|_{p}\right]+\mathbb{E}\left[\left(p-\operatorname{MC}\left(x_{i}\right)\right) \frac{\partial x_{i}}{\partial p} \frac{\partial p}{\partial a}\right] .
$$

Since $\partial p / \partial a=\beta \lambda(\beta+\lambda)^{-1}(\theta-\hat{z})$ and $\partial x_{i} / \partial p=\lambda^{-1}$ we have that

$$
\left.\frac{\partial \mathbb{E}[\mathrm{TS}]}{\partial a}\right|_{a=a_{\text {exo }}^{*}}=\mathbb{E}\left[\left.\left(p-\operatorname{MC}\left(x_{i}\right)\right) \frac{\partial x_{i}}{\partial p} \frac{\partial p}{\partial a}\right|_{a=a_{e x o}^{*}}\right]=\beta(\beta+\lambda)^{-1} \mathbb{E}\left[\left(p-\operatorname{MC}\left(x_{i}\right)\right)(\theta-\hat{z})\right] .
$$

Now, at the market solution

$$
\mathbb{E}\left[\left(p-\operatorname{MC}\left(x_{i}\right)\right)\left(s_{i}-\hat{z}\right)\right]=\mathbb{E}\left[\left(p-\operatorname{MC}\left(x_{i}\right)\right)(\theta-\hat{z})\right]+\mathbb{E}\left[\left(p-\operatorname{MC}\left(x_{i}\right)\right) \varepsilon_{i}\right]=0,
$$

and $\mathbb{E}\left[\left(p-\mathrm{MC}\left(x_{i}\right)\right) \varepsilon_{i}\right]=-\mathbb{E}\left[\mathrm{MC}\left(x_{i}\right) \varepsilon_{i}\right]=-\mathbb{E}\left[\left(\theta+\lambda x_{i}\right) \varepsilon_{i}\right]=\lambda a_{\mathrm{exo}}^{*} \sigma_{\varepsilon}^{2}>0$. The result follows since $\mathbb{E}\left[\left(p-\mathrm{MC}\left(x_{i}\right)\right)(\theta-\hat{z})\right]=-\mathbb{E}\left[\left(p-\mathrm{MC}\left(x_{i}\right)\right) \varepsilon_{i}\right]$.

\section{A.2 Endogenous public information}

Characterization of the equilibrium

The supply function of firm $i$ is given by $X\left(s_{i}, p\right)=\lambda^{-1}\left(p-\mathbb{E}\left[\theta \mid s_{i}, p\right]\right)$, where $p=P(\theta, u)$. For a linear strategy it may be written as

$$
x_{i}=\hat{b}+\hat{c} p-a s_{i}
$$

in which case the aggregate action is given by 


$$
\tilde{x}=\int_{0}^{1} x_{i} d i=\hat{b}+\hat{c} p-a \theta .
$$

It then follows from $p=\alpha+u-\beta \tilde{x}$ that, provided $\hat{c} \neq-\beta^{-1}$,

$$
p=P(\theta, u)=(1+\beta \hat{c})^{-1}(\alpha-\beta \hat{b}+z) ;
$$

here the random variable $z=\kappa \theta+\omega$ has $\kappa=\beta a$ and $\omega=u$, and $z$ is informationally equivalent to the price $p\left(\mathbb{E}\left[\theta \mid s_{i}, p\right]=\mathbb{E}\left[\theta \mid s_{i}, z\right]\right)$. With some abuse of notation, we can posit strategies of the form

$$
X\left(s_{i}, z\right)=b-a s_{i}+c z
$$

and obtain that $p=\alpha-\beta b+(1-\beta c) z$. If $1+\beta \hat{c}>0$ then $1-\beta c>0$ (since $\hat{c}=\left(c^{-1}-\beta\right)^{-1}$ and $\left.1+\beta \hat{c}=(1-\beta c)^{-1}\right)$ and so $p$ and $z$ will move together. The strategy of player $i$ is then given by

$$
X\left(s_{i}, z\right)=\lambda^{-1}\left(\alpha-\beta b+(1-\beta c) z-\mathbb{E}\left[\theta \mid s_{i}, z\right]\right) .
$$

The following expanded Proposition 2 characterizes the equilibrium.

Proposition 2A. Let $\tau_{\varepsilon} \geq 0$ and $\tau_{u} \geq 0$. There is a unique (and symmetric) equilibrium

$$
X\left(s_{i}, p\right)=\lambda^{-1}\left(p-\mathbb{E}\left[\theta \mid s_{i}, p\right]\right)=\hat{b}-a s_{i}+\hat{c} p,
$$

where $a$ is the unique (real) solution of the equation $a=\tau_{\varepsilon} \lambda^{-1}\left(\tau_{\varepsilon}+\tau\right)^{-1}$ with $\tau=\tau_{\theta}+\tau_{u} \beta^{2} a^{2}, \hat{c}=\left((\beta+\lambda)\left(1-\beta \lambda \tau_{u} a^{2} \tau_{\varepsilon}^{-1}\right)^{-1}-\beta\right)^{-1}$, and $\hat{b}=\alpha(1-\lambda \hat{c}) /(\beta+\lambda)$.

Corollary: Let $\tau_{\varepsilon}>0$ and $\tau_{u}>0$. In equilibrium,

- $\quad a \in\left(0, \tau_{\varepsilon} \lambda^{-1}\left(\tau_{\theta}+\tau_{\varepsilon}\right)^{-1}\right)$ decreases with $\tau_{u}, \tau_{\theta},|\beta|$ and $\lambda$, and increases with $\tau_{\varepsilon}$;

- $\operatorname{sgn}\left\{\partial \hat{c} / \partial \tau_{u}\right\}=\operatorname{sgn}\left\{-\partial \hat{c} / \partial \tau_{\theta}\right\}=\operatorname{sgn}\{-\beta\} \quad, \quad$ and market depth $(\partial P / \partial u)^{-1}=1+\beta \hat{c}>0$ is decreasing in $\tau_{u}$ and increasing in $\tau_{\theta}$; and

- price informativeness $\tau$ is increasing in $|\beta|, \tau_{u}, \tau_{\theta}$ and $\tau_{\varepsilon}$, and decreasing in $\lambda$.

Remark: With $\beta>0$, as $\tau_{u}$ increases from $0, \hat{c}$ decreases from $\lambda^{-1}$. As $\tau_{u}$ tends to $\infty, \hat{c}$ tends to $-\beta^{-1}$. When $\beta<0$ increasing $\tau_{u}$, which reinforces the informational component of the price, increases $\hat{c}$ - the opposite of what happens when $\tau_{\theta}$ increases. It follows that in either case $(\beta>0$ or $\beta<0)$ market depth 
$(\partial P / \partial u)^{-1}=1+\beta \hat{c}$ is decreasing in $\tau_{u}$ and increasing in $\tau_{\theta}$. (See the online appendix for more comparative statics properties of the equilibrium.)

Proof of Proposition 2A: From the posited strategy $X\left(s_{i}, z\right)=b-a s_{i}+c z$, where $z=u+\beta a \theta$ and $1-\beta c \neq 0$, we obtain that $p=\alpha-\beta b+(1-\beta c) z$. From the firstorder condition for player $i$ we have

$$
X\left(s_{i}, z\right)=\lambda^{-1}\left(\alpha-\beta b+(1-\beta c) z-\mathbb{E}\left[\theta \mid s_{i}, z\right]\right) .
$$

Here $\mathbb{E}\left[\theta \mid s_{i}, z\right]=\gamma s_{i}+(1-\gamma) \mathbb{E}[\theta \mid z]$ with $\gamma=\tau_{\varepsilon}\left(\tau_{\varepsilon}+\tau\right)^{-1}, \quad \mathbb{E}[\theta \mid z]=\beta \tau_{u} a \tau^{-1} z$ (recall that we have normalized $\bar{\theta}=0$ ), and $\tau=\tau_{\theta}+\beta^{2} a^{2} \tau_{u}$ from the projection theorem for Gaussian random variables. Note that $\mathbb{E}\left[\theta \mid s_{i}, z\right]=\gamma s_{i}+h z$ where $h=\beta a \tau_{u}\left(\tau_{\varepsilon}+\tau\right)^{-1}$. Identifying coefficients with $X\left(s_{i}, z\right)=b-a s_{i}+c z$, we can immediately obtain

$$
a=\frac{\gamma}{\lambda}=\frac{\tau_{\varepsilon}}{\lambda\left(\tau_{\varepsilon}+\tau\right)}, \quad c=\frac{1-h}{\beta+\lambda}=\frac{1}{\beta+\lambda}-\frac{\beta a \tau_{u}}{(\beta+\lambda)\left(\tau_{\varepsilon}+\tau\right)}, \quad \text { and } \quad b=\frac{\alpha}{\beta+\lambda} .
$$

It follows that the equilibrium parameter $a$ is determined as the unique (real), of the following cubic equations, that is positive and lies in the interval $a \in\left(0, \tau_{\varepsilon} \lambda^{-1}\left(\tau_{\theta}+\tau_{\varepsilon}\right)^{-1}\right):$

$$
a=\frac{\tau_{\varepsilon}}{\lambda\left(\tau_{\varepsilon}+\tau_{\theta}+\beta^{2} a^{2} \tau_{u}\right)} \quad \text { or } \quad \beta^{2} \tau_{u} a^{3}+\left(\tau_{\varepsilon}+\tau_{\theta}\right) a-\lambda^{-1} \tau_{\varepsilon}=0
$$

and

$$
c=\frac{1}{(\beta+\lambda)}-\frac{\beta \lambda \tau_{u} a^{2}}{(\beta+\lambda) \tau_{\varepsilon}}
$$

It is immediate from the preceding equality for $c$ that $c<(\beta+\lambda)^{-1}$ (since $a \geq 0$ ) and that $1-\beta c>0$ (since $\beta+\lambda>0$ ); therefore,

$$
\beta c=\frac{\beta}{\beta+\lambda}-\frac{\beta^{2} a \tau_{u}}{(\beta+\lambda)\left(\tau_{\varepsilon}+\tau\right)}<1
$$

It follows that

$$
X\left(s_{i}, p\right)=\hat{b}-a s_{i}+\hat{c} p,
$$


where $\hat{b}=b(1-\lambda \hat{c}), b=\alpha /(\beta+\lambda)$, and $\hat{c}=c /(1-\beta c)=\left(c^{-1}-\beta\right)^{-1}$ with $1+\beta \hat{c}>0$.

See the online appendix for the proof of the Corollary.

\section{Characterization of the team solution}

Claim 2: The strategy at the team solution is of the form $X\left(s_{i}, z ; p\right)=\lambda^{-1}\left(p-\left(\gamma s_{i}+(1-\gamma) \hat{z}\right)\right)$ where $\hat{z} \equiv \mathbb{E}[\theta \mid z]$.

Proof: The team solution solves program ( $\mathbf{T}$ ). It can be checked that $\partial^{2} \mathbb{E}[\mathrm{TS}] / \partial^{2} b<0$ and $\partial^{2} \mathbb{E}[\mathrm{TS}] / \partial^{2} c<0$ whenever $\beta+\lambda>0$. Given that $\partial x_{i} / \partial b=1$, and $\partial x_{i} / \partial c=z$, we can optimize with respect to $b$ and $c$ to obtain

$$
\begin{aligned}
& \frac{\partial \mathbb{E}[\mathrm{TS}]}{\partial b}=\mathbb{E}\left[\left(p-\operatorname{MC}\left(x_{i}\right)\right)\right]=0, \\
& \frac{\partial \mathbb{E}[\mathrm{TS}]}{\partial c}=\mathbb{E}\left[\left(p-\operatorname{MC}\left(x_{i}\right)\right) z\right]=0,
\end{aligned}
$$

where $p=\alpha+u-\beta \tilde{x}$ and $\operatorname{MC}\left(x_{i}\right)=\theta+\lambda x_{i}$. The constraint $\mathbb{E}\left[p-\operatorname{MC}\left(x_{i}\right)\right]=0$ can be seen equivalent to $b=\alpha /(\beta+\lambda)$, and $\mathbb{E}\left[\left(p-\operatorname{MC}\left(x_{i}\right)\right) z\right]=0$ to $c=c(a) \equiv \frac{1}{\beta+\lambda}-\frac{\beta a \tau_{u}(1-\lambda a)}{\tau(\beta+\lambda)}$. Those constraints are also fulfilled by the market solution since the first-order condition (FOC) for playeri is $\mathbb{E}\left[p-\operatorname{MC}\left(x_{i}\right) \mid s_{i}, z\right]=0$, from which it follows, according to the properties of Gaussian distributions, that $\mathbb{E}\left[p-\operatorname{MC}\left(x_{i}\right)\right]=0$, and $\mathbb{E}\left[\left(p-\operatorname{MC}\left(x_{i}\right)\right) z\right]=0$ (as well as $\mathbb{E}\left[\left(p-\operatorname{MC}\left(x_{i}\right)\right) s_{i}\right]=0$, which is equivalent to $\left.c=\frac{a\left(\lambda\left(\tau_{\varepsilon}+\tau_{\theta}\right)+\beta \tau_{\varepsilon}\right)-\tau_{\varepsilon}}{a \beta \tau_{\varepsilon}(\lambda+\beta)}\right)$. Using the expressions for $b=\alpha /(\beta+\lambda)$ and $c=\frac{1}{\beta+\lambda}-\frac{\beta a \tau_{u}(1-\lambda a)}{\tau(\beta+\lambda)}$, using the fact that $\mathbb{E}[\theta \mid z]=\beta \tau_{u} a \tau^{-1} z$ and $\tilde{x}=b-a \theta+c z$, we find that $x_{i}=\lambda^{-1}\left(p-\left(\gamma s_{i}+(1-\gamma) \mathbb{E}[\theta \mid z]\right)\right)$ where $\gamma=\lambda a$.

From the FOC in the minimization ofWL, $d \mathrm{WL}(a ; \tau(a)) / d a=0$ with 


$$
\frac{\partial \mathrm{WL}}{\partial a}=\frac{\lambda a}{\tau_{\varepsilon}}-\frac{\lambda(1-\lambda a)}{(\beta+\lambda) \tau} \text { and } \frac{\partial \mathrm{WL}}{\partial \tau} \frac{\partial \tau}{\partial a}=-\frac{(1-\lambda a)^{2} \beta^{2} a \tau_{u}}{(\beta+\lambda) \tau^{2}}
$$

we obtain that $a^{T}$ fulfills

$$
a=\frac{\tau_{\varepsilon}}{\lambda\left(\tau(a)+\tau_{\varepsilon}\right)+\beta \tau(a)-\Delta(a)}
$$

where $\beta \tau(a)$ corresponds to the pecuniary externality and $\Delta(a)=\frac{(1-\lambda a)^{2} \beta^{2} \tau_{u} \tau_{\varepsilon}}{\lambda \tau(a)} \geq 0$ to the learning externality.

Claim 3: When $\beta>0$, at the market solution $\operatorname{sgn}\{\beta \tau-\Delta\}=\operatorname{sgn}\left\{c^{*}\right\}$.

Proof: When at the market solution we have that $c^{*}=0$ then $\beta \tau=\Delta$. This is so since we can check that $\Delta=\frac{\tau \tau_{\varepsilon}(1-(\beta+\lambda) c)^{2}}{\lambda \tau_{u} a^{2}}$ and therefore $\beta \tau=\Delta$ is equivalent to $\beta=\frac{\tau_{\varepsilon}}{\lambda \tau_{u} a^{2}}$ when $c=0$. The result follows since at the market equilibrium $c=(\beta+\lambda)^{-1}\left(1-\beta \lambda \tau_{u} a^{2} \tau_{\varepsilon}^{-1}\right)$ (from Proposition 2A) and therefore $1=\beta \lambda \tau_{u} a^{2} \tau_{\varepsilon}^{-1}$ when $c^{*}=0$. At the market solution, when $c^{*}>0$ we have that $\beta \tau-\Delta>0$ and when $c^{*}<0$ we have that $\beta \tau-\Delta<0$. This is immediate since at the market solution $\frac{\tau_{\varepsilon}(1-(\beta+\lambda) c)^{2}}{\lambda \tau_{u} a^{2}}=\beta(1-(\beta+\lambda) c)$

From the Lemma (Section 4.2) we obtain the effect of the pecuniary externality for given public information at the market solution $a^{*}$ :

$$
\mathbb{E}\left[\left.\left(p-\operatorname{MC}\left(x_{i}\right)\right) \frac{\partial x_{i}}{\partial p} \frac{\partial p}{\partial a}\right|_{\hat{z} \text { ct. }}\right]=-\beta \lambda(\beta+\lambda)^{-1} a^{*} \sigma_{\varepsilon}^{2} .
$$

Furthermore, the learning externality has the expected sign

$$
\mathbb{E}\left[\left(p-\operatorname{MC}\left(x_{i}\right)\right)\left(\frac{\partial x_{i}}{\partial \hat{z}} \frac{\partial \hat{z}}{\partial a}\right)\right]=\frac{1-\lambda a^{*}}{\beta+\lambda} \frac{\beta^{2} \tau_{u} a^{*}}{\tau^{*}}\left(\lambda a^{*} \sigma_{\varepsilon}^{2}\right)>0
$$

and adding up both externality effects delivers the desired result $\operatorname{sgn}\{\partial \mathbb{E}[\mathrm{TS}] / \partial a\}=\operatorname{sgn}\left\{-\beta c^{*}\right\} \quad$ (using the fact that $c^{*}=(\beta+\lambda)^{-1}\left(1-\beta \tau_{u} a^{*}\left(\tau_{\varepsilon}+\tau^{*}\right)^{-1}\right)$ from the proof of Proposition 2A). 
Proof of Proposition 4. The welfare loss at the team-efficient solution is given by $\mathrm{WL}\left(a^{\mathrm{T}}\right)$, which is decreasing in $\tau_{\varepsilon}, \tau_{\omega}, \tau_{u}$ and $\tau_{\theta}$ since $\mathrm{WL}$ is decreasing in those parameters for a given $a$ and $d \mathrm{WL}\left(a^{\mathrm{T}}\right) / d a=0$. Let $2 \beta+\lambda>0$. With respect to the market solution we have that

$$
\frac{d \mathrm{WL}}{d \tau_{\theta}}\left(a^{*}\right)=\frac{\partial \mathrm{WL}}{\partial a} \frac{\partial a^{*}}{\partial \tau_{\theta}}+\frac{\partial \mathrm{WL}}{\partial \tau_{\theta}},
$$

where $\frac{\partial a^{*}}{\partial \tau_{\theta}}=-\frac{a}{\tau_{\theta}+\tau_{\varepsilon}+3 a^{2} \beta^{2} \tau_{u}}$ and $a^{*}$ solves $\beta^{2} \tau_{u} a^{3}+\left(\tau_{\varepsilon}+\tau_{\theta}\right) a-\lambda^{-1} \tau_{\varepsilon}=0$.

Given that the expression for WL it is possible to show that

$$
\frac{d \mathrm{WL}}{d \tau_{\theta}}\left(a^{*}\right)<0 \text { if and only if } \frac{\tau_{\theta}+\tau_{u} \beta^{2} a^{2}}{\tau_{\varepsilon}}>-\frac{2 \beta+\lambda}{\lambda},
$$

which is always true since $2 \beta+\lambda>0$. Exactly the same condition holds for $d \mathrm{WL}\left(a^{*}\right) / d \tau_{u}<0$. Furthermore, we can show that $d \mathrm{WL}\left(a^{*}\right) / d \tau_{\varepsilon} \leq 0$ if and only if $\beta-\lambda \leq \frac{\tau_{u} \beta^{2} a^{*}}{\tau_{\theta}}\left(a^{*}(\beta+\lambda)+2\right)+(\beta+\lambda) \frac{\tau_{\varepsilon}}{\tau_{\theta}}$. It follows that WL will be increasing in $\tau_{\varepsilon}$ for $\beta>\lambda$ and $\tau_{\varepsilon} / \tau_{\theta}$ small enough (since $a^{*}$ is increasing in $\tau_{\varepsilon} / \tau_{\theta}$ ).

\section{References}

Amador, M. and P.O. Weill (2010), "Learning from Prices: Public Communication and Welfare", Journal of Political Economy, 118, 5, 866-907.

Amador, M. and P.O. Weill (2012), "Learning from Private and Public Observations of Others' Actions", Journal of Economic Theory, 147, 3, 910-940.

Angeletos G. M. and J. La'O (2013), “Optimal Monetary Policy with Informational Frictions", mimeo.

Angeletos G. M., L. Iovino and J. La'O (2015), "Efficiency and Policy with Endogenous Learning”, mimeo.

Angeletos, G. M. and A. Pavan (2007), "Efficient Use of Information and Social Value of Information”, Econometrica, 75, 4, 1103-1142.

Angeletos, G.M. and A. Pavan (2009), "Policy with Dispersed Information", Journal of the European Economic Association, 7, 1, 11-60. 
Bayona, A. (2015), “The Social Value of Information with an Endogenous Public Signal", WP Esade Business school.

Banerjee, A. (1992), “A Simple Model of Herd Behavior”, Quarterly Journal of Economics, 107, 3, 797-819.

Bikhchandani, S., D. Hirshleifer and I. Welch (1992), "A Theory of Fads, Fashion, Custom, and Cultural Change as Informational Cascades”, Journal of Political Economy, 100, 5, 992-1026.

Burguet, R. and X. Vives (2000), "Social Learning and Costly Information Acquisition", Economic Theory, 15, 1,185-205.

Caballero, R. and A. Krishnamurthy (2001), "International Collaterial Constraints in a Model of Emerging Market Crises”, Journal of Monetary Economics, 48, 3, 513548.

Colombo, L, G. Femminis and A. Pavan (2014), "Information Acquisition and Welfare", Review of Economic Studies, 81, 4, 1438-1883.

Dow, J. and R. Rahi (2000), “Should Speculators be Taxed?”, Journal of Business, 73, 1, 89-107.

Eyster, E. and M. Rabin (2005), “Cursed Equilibrium”, Econometrica, 73, 5, 1623 1672.

Gennotte, G. and H. Leland (1990), "Market Liquidity, Hedging, and Crashes", American Economic Review”, 80, 5, 1990, 999-1021.

Greenwald, B. and J. Stiglitz (1986), "Externalities in Economies with Imperfect Information and Incomplete Markets", Quarterly Journal of Economics, 101, 2, 229-264.

Hayek, F. A. (1945), "The Use of Knowledge in Society", American Economic Review, 35, 4, 519-530.

Hellwig, C. (2005), "Heterogeneous Information and the Welfare Effects of Public Information Disclosures”, Mimeo, U.C.L.A.

Hellwig, C. and L. Veldkamp (2009), "Knowing What Others Know: Coordination Motives in Information Acquisition", Review of Economic Studies, 76, 223-251. 
Hendershott, T., and M. Seasholes (2009), "Market Predictability and NonInformational Trading”, mimeo.

Hirshleifer, J. (1971), “The Private and Social Value of Information and the Reward to Inventive Activity", American Economic Review, 61, 4, 561-574.

Jeanne, O. and A. Korinek (2010), "Excessive Volatility in Capital Flows: A Pigouvian Taxation Approach", American Economic Review, 100, 403-407.

Keynes, J. M. (1936), The General Theory of Employment Interest and Money, Palgrave MacMillan.

Kyle, A. (1985), "Continuous Auctions and Insider Trading”, Econometrica, 53, $1315-1335$.

Laffont, J.J. (1985), “On the Welfare Analysis of Rational Expectations Equilibria with Asymmetric Information", Econometrica, 53, 1-29.

Llosa, L.G., and V. Venkateswaran (2013), "Efficiency with Endogenous Information Choice", mimeo.

Lorenzoni, G. (2010), “Optimal Monetary Policy with Uncertain Fundamentals and Dispersed Information”, Review of Economic Studies, 77, 1, 305-338.

Messner, S. and X. Vives (2006), "Informational and Economic Efficiency in REE with Asymmetric Information"; mimeo.

Morris, S. and H. Shin (2002), "The Social Value of Public Information”, American Economic Review, 92, 1521-1534.

Morris, S. and H.S. Shin (2005), "Central Bank Transparency and the Signal Value of Prices", Brookings Papers on Economic Activity, 2, 43-85.

Myatt, D. and C. Wallace (2012), "Endogenous Information Acquisition in Coordination Games", Review of Economic Studies, 79, 1, 340-374.

Palfrey, T. R. (1985), “Uncertainty Resolution, Private Information Aggregation and the Cournot Competitive Limit”, Review of Economic Studies, 52, 69-83.

Radner, R. (1979), "Rational Expectations Equilibrium: Generic Existence and the Information Revealed by Prices", Econometrica, 49, 655-78.

Schlee, E. (2001), "The Value of Information in Efficient Risk-Sharing Arrangements", American Economic Review, 91,3, 509-524. 
Subrahmanyam, A. (1998), "Transaction Taxes and Financial Market Equilibrium", Journal of Business, 71, 1, 81-118.

Stiglitz, J. (1989), "Using Tax Policy to Curb Speculative Short-term Trading", Journal of Financial Services Research, 3, 101-115.

Summers, L., and V. Summers (1989), "When Financial Markets Work Too Well: A Cautious Case for a Securities Transactions Tax," Journal of Financial Services Research, 3, 261-286.

Tobin, J. (1978), “A Proposal for International Monetary Reform”, Eastern Economic Journal, 4, 153-159.

Vives, X. (1988), "Aggregation of Information in Large Cournot Markets", Econometrica, 56, 4, 851-876.

Vives, X. (1993), “How Fast Do Rational Agents Learn?", Review of Economic Studies, 60, 2, 329-347.

Vives, X. (1997), "Learning from Others: A Welfare Analysis”, Games and Economic Behavior, 20, 2, 177-200.

Vives, X. (2008), Information and Learning in Markets, Princeton: Princeton University Press.

Vives , X. (2011), “Strategic Supply Function Competition with Private Information”, Econometrica, 79, 6, 1919-1966.

Vives (2014), "On the Possibility of Informationally Efficient Markets", Journal of the European Economic Association, 12, 5, 1200-1239. 


\section{Online Appendix to}

\section{Endogenous Public Information and Welfare in Market Games}

\section{Xavier Vives}

\section{Suplement to the equilibrium characterization of Section 5.1}

Claim A. Linear equilibria in strategies with bounded means and with uniformly (across players) bounded variances yield linear equilibria of the schedule game for which the public statistic function is of type $P(\theta, u)$.

Proof: If for player $i$ we posit the strategy

$$
x_{i}=\hat{b}_{i}+\hat{c}_{i} p-a_{i} s_{i}
$$

then the aggregate action is given by

$$
\tilde{x}=\int_{0}^{1} x_{i} d i=\hat{b}+\hat{c} p-a \theta-\int_{0}^{1} a_{i} \varepsilon_{i} d i=\hat{b}+\hat{c} p-a \theta,
$$

where $\hat{b}=\int_{0}^{1} \hat{b}_{i} d i, \hat{c}=\int_{0}^{1} \hat{c}_{i} d i$, and $a=\int_{0}^{1} a_{i} d i$ (assuming that all terms are welldefined). Observe that, according to our convention on the average error terms of the signals, $\int_{0}^{1} a_{i} \varepsilon_{i} d i=0$ a.s. provided that $\operatorname{var}\left[a_{i} \varepsilon_{i}\right]$ is uniformly bounded across agents (since $\operatorname{var}\left[\varepsilon_{i}\right]=\sigma_{\varepsilon}^{2}$, it is enough that $a_{i}$ be uniformly bounded). In equilibrium, this will be the case. Therefore, if we restrict attention to candidate linear equilibria with parameters $a_{i}$ uniformly bounded in $i$ and with well-defined average parameters $\hat{b}$ and $\hat{c}$, then $\tilde{x}=\hat{b}+\hat{c} p-a \theta$ and the public statistic function is of the type $P(\theta, u)$.

\section{Proof of Corollary to Proposition $2 \mathrm{~A}$}

(i) From the equation determining the responsiveness to private information $a$, $\beta^{2} \tau_{u} a^{3}+\left(\tau_{\varepsilon}+\tau_{\theta}\right) a-\lambda^{-1} \tau_{\varepsilon}=0$, it is immediate that $a$ decreases with $\tau_{u}, \tau_{\theta}, \beta^{2}$ and $\lambda$, that $a$ increases with $\tau_{\varepsilon}$. Note that $\operatorname{sgn}\{\partial a / \partial \beta\}=\operatorname{sgn}\{-\beta\}$. As $\tau_{u}$ ranges from 0 to $\infty, a$ decreases from $\lambda^{-1} \tau_{\varepsilon}\left(\tau_{\theta}+\tau_{\varepsilon}\right)^{-1}$ to 0 . 
(ii) As $\tau_{u}$ ranges from 0 to $\infty$, the responsiveness to public information $c$ goes from $(\beta+\lambda)^{-1}$ to $-\infty$ (resp. $\left.+\infty\right)$ if $\beta>0$ (resp. $\left.\beta<0\right)$. The result follows since, in equilibrium,

$$
c=\frac{1}{\beta+\lambda}-\frac{\beta \lambda \tau_{u} a^{2}}{(\beta+\lambda) \tau_{\varepsilon}}=\frac{1}{\beta+\lambda}-\frac{1}{(\beta+\lambda) \beta}\left(\frac{1}{a}-\lambda\left(1+\frac{\tau_{\theta}}{\tau_{\varepsilon}}\right)\right)
$$

and $a \rightarrow 0$ as $\tau_{u} \rightarrow \infty$. It follows that $\operatorname{sgn}\left\{\partial c / \partial \tau_{u}\right\}=\operatorname{sgn}\{-\beta\}$ because $\partial a / \partial \tau_{u}<0$. Similarly, from the first part of the expression for $c$ we have $\operatorname{sgn}\left\{\partial c / \partial \tau_{\theta}\right\}=\operatorname{sgn}\{\beta\}$ since $\partial a / \partial \tau_{\theta}<0$. Since $\hat{c}=\left(c^{-1}-\beta\right)^{-1}$, it follows that $\hat{c}$ goes from $\lambda^{-1}$ to $-\beta^{-1}$ as $\tau_{u} \quad$ ranges from 0 to $\infty, 1 \quad \operatorname{sgn}\left\{\partial \hat{c} / \partial \tau_{u}\right\}=\operatorname{sgn}\left\{-\partial \hat{c} / \partial \tau_{\theta}\right\}=\operatorname{sgn}\{-\beta\}$, and $\operatorname{sgn}\left\{\partial \hat{c} / \partial \tau_{\varepsilon}\right\}=\operatorname{sgn}\left\{\partial c / \partial \tau_{\varepsilon}\right\}$. It is then immediate that $1+\beta \hat{c}$ is decreasing in $\tau_{u}$ and increasing in $\tau_{\theta}$.

(iii) Price informativeness $\tau=\tau_{\theta}+\beta^{2} a^{2} \tau_{u}$ is increasing in $\tau_{\varepsilon}$ (since $a$ increases with $\tau_{\varepsilon}$ ) and also in $\tau_{u}$ (since $a=\lambda^{-1} \tau_{\varepsilon}\left(\tau_{\varepsilon}+\tau\right)^{-1}$ and $a$ decreases with $\tau_{u}$ ). Using the expression for $\partial a / \partial \tau_{\theta}$ we have that

$$
\frac{\partial \tau}{\partial \tau_{\theta}}=1+2 \beta^{2} \tau_{u} a \frac{\partial a}{\partial \tau_{\theta}}=1-\frac{2 \beta^{2} a^{2} \tau_{u}}{\tau_{\theta}+\tau_{\varepsilon}+3 a^{2} \beta^{2} \tau_{u}}=\frac{\tau_{\theta}+\tau_{\varepsilon}+a^{2} \beta^{2} \tau_{u}}{\tau_{\theta}+\tau_{\varepsilon}+3 a^{2} \beta^{2} \tau_{u}}>0
$$

Furthermore,

$$
\frac{\partial \tau}{\partial \beta}=\tau_{u}\left(2 \beta a^{2}+2 \beta^{2} a \frac{\partial a}{\partial \beta}\right)=2 \beta a \tau_{u}\left(a-\frac{2 a^{4} \tau_{u} \lambda \beta^{2}}{1+2 a^{3} \tau_{u} \lambda \beta^{2}}\right)=\frac{2 \beta a^{2} \tau_{u}}{1+2 a^{3} \tau_{u} \lambda \beta^{2}}
$$

and therefore $\tau$ increases in $|\beta|$.

\section{Proof of Proposition 5 (internal team solution)}

Let $2 \beta+\lambda>0$. The proof proceeds in a parallel way to the proof of Proposition 3. It can be checked first that $\partial^{2} \mathbb{E}\left[\pi_{i}\right] / \partial^{2} b<0$ and $\partial^{2} \mathbb{E}\left[\pi_{i}\right] / \partial^{2} c<0$ whenever $2 \beta+\lambda>0$.

1 Note that if $\beta<0$ and $\beta+\lambda>0$ then $\lambda^{-1}<-\beta^{-1}$. 
Given that $\pi_{i}=p x_{i}-\mathrm{C}\left(x_{i}\right), p=\alpha+u-\beta \tilde{x}, \partial x_{i} / \partial b=1$, and $\partial x_{i} / \partial c=\partial \tilde{x} / \partial c=z$ and $\partial p / \partial \tilde{x}=-\beta$ we can optimize with respect to $b$ and $c$ to obtain

$$
\begin{aligned}
& \frac{\partial \mathbb{E}\left[\pi_{i}\right]}{\partial b}=\mathbb{E}\left[\left(p-\operatorname{MC}\left(x_{i}\right)\right)-\beta x_{i}\right]=0, \\
& \frac{\partial \mathbb{E}\left[\pi_{i}\right]}{\partial c}=\mathbb{E}\left[\left(p-\operatorname{MC}\left(x_{i}\right)\right) z-\beta x_{i} z\right]=0 .
\end{aligned}
$$

where $\operatorname{MC}\left(x_{i}\right)=\theta+\lambda x_{i}$. The constraint $\mathbb{E}\left[\left(p-\operatorname{MC}\left(x_{i}\right)\right)-\beta x_{i}\right]=0$ is equivalent to $b=\alpha /(2 \beta+\lambda)$; we can also check that $\mathbb{E}\left[\left(p-\operatorname{MC}\left(x_{i}\right)\right) z-\beta x_{i} z\right]=0$ is equivalent to $c=c^{\mathrm{IT}}(a)$, where

$$
c^{\mathrm{IT}}(a)=\frac{1}{2 \beta+\lambda}-\frac{\beta a \tau_{u}(1-(\lambda+\beta) a)}{\tau(2 \beta+\lambda)} \quad \text { and } \quad \tau=\tau_{\theta}+\beta^{2} \tau_{u} a^{2} .
$$

Note that due to the competition payoff externality $(\partial p / \partial \tilde{x}=-\beta)$ the expressions for $b$ and for $c$ are different than in the market solution. It follows that the form of the internal team optimal strategy is $x_{i}=(\lambda+\beta)^{-1}\left(p-\left(\gamma s_{i}+(1-\gamma) \mathbb{E}[\theta \mid z]\right)\right)$ where $\gamma=(\lambda+\beta) a$. We have that $\tilde{x}=(\lambda+\beta)^{-1}(p-(\gamma \theta+(1-\gamma) \mathbb{E}[\theta \mid z]))$ and that $\tilde{x}-x^{\mathrm{M}}=(1-\gamma)(\theta-\mathbb{E}[\theta \mid z]) /(2 \beta+\lambda) \quad$ and, since $\tau=(\operatorname{var}[\theta \mid z])^{-1} \quad$ we obtain $\mathbb{E}\left[\left(\tilde{x}-x^{\mathrm{M}}\right)^{2}\right]=(1-(\lambda+\beta) a)^{2} /\left(\tau(2 \beta+\lambda)^{2}\right)$. We have that $\mathbb{E}\left[\left(x_{i}-\tilde{x}\right)^{2}\right]=a^{2} / \tau_{\varepsilon}$.

Let $\Omega=\mathbb{E}\left[\pi_{i}^{\mathrm{M}}\right]-\mathbb{E}\left[\pi_{i}\right]$. Similarly as before we can obtain that $\Omega=\left((2 \beta+\lambda) \mathbb{E}\left[\left(\tilde{x}-x^{\mathrm{M}}\right)^{2}\right]+\lambda \mathbb{E}\left[\left(x_{i}-\tilde{x}\right)^{2}\right]\right) / 2$. It follows that

$$
\Omega(a)=\frac{1}{2}\left(\frac{(1-(\lambda+\beta) a)^{2}}{\left(\tau_{\theta}+\tau_{u} \beta^{2} a^{2}\right)(2 \beta+\lambda)}+\frac{\lambda a^{2}}{\tau_{\varepsilon}}\right),
$$

which is easily seen strictly convex in $a$ and with a unique solution $(\lambda+\beta)^{-1}>a^{\mathrm{IT}}>0$. (Note that $(\lambda+\beta)^{-1}<a$ is dominated by $a=(\lambda+\beta)^{-1}$ and that $a<0$ is dominated by $-a>0$. Furthermore, it is immediate that $\Omega^{\prime}(0)<0$ and therefore $a>0$ at the solution.) 
The impact of $a$ on $\mathbb{E}\left[\pi_{i}\right]$ is easily characterized (noting that $\partial \mathbb{E}\left[\pi_{i}\right] / \partial c=0$ and therefore disregarding the indirect impact of $a$ on $\mathbb{E}\left[\pi_{i}\right]$ via a change in $c$ ):

$$
\begin{aligned}
\frac{\partial \mathbb{E}\left[\pi_{i}\right]}{\partial a}= & \underbrace{\mathbb{E}\left[\left(p-M C\left(x_{i}\right)\right)\left(\frac{\partial x_{i}}{\partial a}\right)_{z c t .}\right]_{\text {Market }}}+ \\
& \underbrace{\mathbb{E}\left[\left(p-M C\left(x_{i}\right)\right)\left(\frac{\partial x_{i}}{\partial z} \frac{\partial z}{\partial a}\right)\right]}+ \\
& \underbrace{\left[x_{i}\left(\frac{\partial p}{\partial \tilde{x}} \frac{\partial \tilde{x}}{\partial a}\right)\right]}_{\mathrm{PE}+\mathrm{LE}} \underbrace{}_{\mathrm{CE}} \\
& =\mathbb{E}\left[\left(p-\operatorname{MC}\left(x_{i}\right)\right)\left(-s_{i}+c \beta \theta\right)-\beta(c \beta-1) \theta x_{i}\right]
\end{aligned}
$$

given that $\left(\partial x_{i} / \partial a\right)_{z \text { ct. }}=-s_{i}, \quad \partial x_{i} / \partial z=c, \partial z / \partial a=\beta \theta \quad, \quad \partial p / \partial \tilde{x}=-\beta$ and $\partial \tilde{x} / \partial a=(c \beta-1) \theta$. Evaluating $\partial \mathbb{E}\left[\pi_{i}\right] / \partial a$ at the market solution, where $\mathbb{E}\left[\left(p-\operatorname{MC}\left(x_{i}\right)\right) s_{i}\right]=0$, we obtain

$$
\frac{\partial \mathbb{E}\left[\pi_{i}\right]}{\partial a}=\beta \mathbb{E}\left[c\left(p-\operatorname{MC}\left(x_{i}\right)\right) \theta-(c \beta-1) \theta x_{i}\right] .
$$

We know that $\mathbb{E}\left[\left(p-\operatorname{MC}\left(x_{i}\right)\right) \theta\right]=-\lambda a \sigma_{\varepsilon}^{2}<0$ and, recalling that $\bar{\theta}=0$, it is easily checked that $\mathbb{E}\left[\theta x_{i}\right]=a \sigma_{\theta}^{2}(c \beta-1)$. At the equilibrium we have therefore ${ }^{2}$

$$
\frac{\partial \mathbb{E}\left[\pi_{i}\right]}{\partial a}=-\beta a^{*}\left(c^{*} \lambda \sigma_{\varepsilon}^{2}+\left(c^{*} \beta-1\right)^{2} \sigma_{\theta}^{2}\right) \text {. }
$$

Since $\mathbb{E}\left[\pi_{i}\right]$ is single-peaked for $a>0$ and has a unique maximum at $a^{\mathrm{IT}}>0$ and $a^{*}>0$, it follows that $\operatorname{sgn}\left\{a^{\mathrm{IT}}-a^{*}\right\}=\operatorname{sgn}\left\{\left.\frac{\partial \mathbb{E}\left[\pi_{i}\right]}{\partial a}\right|_{a=a^{*}}\right\}=$ $\operatorname{sgn}\left\{-\beta\left(c^{*} \lambda \sigma_{\varepsilon}^{2}+\left(c^{*} \beta-1\right)^{2} \sigma_{\theta}^{2}\right)\right\}$

\section{Efficiency in the Cournot market with endogenous public signals}

In this section we assume that firms compete in quantities. We have shown in Section 4.3 that with exogenous public information the market solution is efficient. Suppose now that public signal $z$ comes from an endogenous noisy quantity signal, $q=\tilde{x}+\eta$

2 Note also that at the equilibrium $c \beta-1<0$. 
where $\eta \sim N\left(0, \tau_{\eta}^{-1}\right)$ is independent of the other random variables in the model. Then positing that firms use a linear strategy $x_{i}=b-a s_{i}-\hat{c} q$ it is easily seen that $q=(1+\hat{c})^{-1}(b-z)$ where $z=a \theta-\eta$. Letting $\hat{z}=\mathbb{E}[\theta \mid z], \quad$ the strategy $X\left(s_{i}, z\right)$ has the same form as before but now $\tau=\tau_{\theta}+\tau_{\eta} a^{2}$ is endogenous.

We may conjecture that the endogenous quantity signal will lead firms to put too little weight on their private information due to the presence of an information externality. We confirm that this is indeed the case. It can be checked that candidate team strategies are of the same form as the market but with potentially a different response $a$ to private information. We have that:

$$
\frac{\partial \mathbb{E}[\mathrm{TS}]}{\partial a}=\mathbb{E}\left[\left.\left(p-\operatorname{MC}\left(x_{i}\right)\right) \frac{\partial x_{i}}{\partial a}\right|_{\hat{z} \text { ct. }}\right]+\mathbb{E}\left[\left(p-\operatorname{MC}\left(x_{i}\right)\right)\left(\frac{\partial x_{i}}{\partial \hat{z}} \frac{\partial \hat{z}}{\partial a}\right)\right] .
$$

At the (Cournot) market solution $\mathbb{E}\left[\left.\left(p-\mathrm{MC}\left(x_{i}\right)\right) \frac{\partial x_{i}}{\partial a}\right|_{z_{\text {ct. }}}\right]=0$ since firms take $z$ as given and the learning externality term is positive, $\mathbb{E}\left[\left(p-\operatorname{MC}\left(x_{i}\right)\right)\left(\frac{\partial x_{i}}{\partial \hat{z}} \frac{\partial \hat{z}}{\partial a}\right)\right]>0$, and therefore $\partial \mathbb{E}[\mathrm{TS}] / \partial a>0$. This indicates that $a$ has to be increased from the market level and, since $\mathbb{E}[\mathrm{TS}]$ is strictly concave in $a$, we conclude as expected that the information externality leads to a too small response to private information. We confirm in Lemma A1 that this is indeed the case.

Lemma A1 (Cournot): Consider the Cournot model of Section 4.3 with the information set of firms augmented with a noisy quantity signal. Let $\tau_{\varepsilon}>0$, then the market solution has a smaller response to private information than the team solution.

Proof: It can be checked that candidate team strategies are of the same form as the market $X\left(s_{i}, z\right)=\alpha(\beta+\lambda)^{-1}-\left(a s_{i}+\left((\beta+\lambda)^{-1}-a\right) \hat{z}\right)$ but with potentially a different response $a$ to private information. We have that: 


$$
\begin{aligned}
\frac{\partial \mathbb{E}[\mathrm{TS}]}{\partial a} & =\mathbb{E}\left[\left.\left(p-\operatorname{MC}\left(x_{i}\right)\right) \frac{\partial x_{i}}{\partial a}\right|_{\hat{z}}\right]+\mathbb{E}\left[\left(p-\operatorname{MC}\left(x_{i}\right)\right)\left(\frac{\partial x_{i}}{\partial \hat{z}} \frac{\partial \hat{z}}{\partial a}\right)\right] \\
& =\mathbb{E}\left[\left(p-\operatorname{MC}\left(x_{i}\right)\right)\left(-\left(s_{i}-\hat{z}\right)-\left((\beta+\lambda)^{-1}-a\right)\left(\tau_{\eta} a \tau^{-1} \theta+z \frac{\partial\left(\tau_{\eta} a \tau^{-1}\right)}{\partial a}\right)\right)\right]
\end{aligned}
$$

since $\hat{z}=\mathbb{E}[\theta \mid z]=\tau_{\eta} a \tau^{-1} z \quad, \quad z=a \theta-\eta \quad$. At the market solution $\mathbb{E}\left[\left.\left(p-\operatorname{MC}\left(x_{i}\right)\right) \frac{\partial x_{i}}{\partial a}\right|_{\hat{z}}\right]=\mathbb{E}\left[\left(p-\operatorname{MC}\left(x_{i}\right)\right)\left(s_{i}-\hat{z}\right)\right]=0$ since firms take $z$ as given, $\mathbb{E}\left[\left(p-\operatorname{MC}\left(x_{i}\right)\right) \hat{z}\right]=\mathbb{E}\left[\left(p-\operatorname{MC}\left(x_{i}\right)\right) z\right]=0$. We have that at the market solution $0<a<(\beta+\lambda)^{-1}$ since $\tau_{\varepsilon}>0$, and

$$
\mathbb{E}\left[\left(p-\operatorname{MC}\left(x_{i}\right)\right)\left(s_{i}-\hat{z}\right)\right]=\mathbb{E}\left[\left(p-\operatorname{MC}\left(x_{i}\right)\right)(\theta-\hat{z})\right]+\mathbb{E}\left[\left(p-\operatorname{MC}\left(x_{i}\right)\right) \varepsilon_{i}\right]=0 .
$$

Therefore,

$$
\begin{aligned}
\mathbb{E}\left[\left(p-\operatorname{MC}\left(x_{i}\right)\right)(\theta-\hat{z})\right] & =\mathbb{E}\left[\left(p-\operatorname{MC}\left(x_{i}\right)\right) \theta\right]=-\mathbb{E}\left[\left(p-\operatorname{MC}\left(x_{i}\right)\right) \varepsilon_{i}\right] \\
& =\mathbb{E}\left[\operatorname{MC}\left(x_{i}\right) \varepsilon_{i}\right]=\mathbb{E}\left[\left(\theta+\lambda x_{i}\right) \varepsilon_{i}\right]=-\lambda a \sigma_{\varepsilon}^{2}<0
\end{aligned}
$$

since $\varepsilon_{i}$ is independent of $\theta$. We conclude that $\mathbb{E}\left[\left(p-\mathrm{MC}\left(x_{i}\right)\right) \theta\right]=-\lambda a \sigma_{\varepsilon}^{2}$ and therefore $\partial \mathbb{E}[\mathrm{TS}] / \partial a=\lambda \tau_{\varepsilon}^{-1}\left((\beta+\lambda)^{-1}-a\right) \tau_{\eta} a^{2} \tau^{-1}>0$. Furthermore, it can be checked that $\mathbb{E}[\mathrm{TS}]$ is strictly concave in $a$ and we can conclude that the team solution calls for a larger response to private information than the market. 